Homology, Homotopy and Applications, vol. 19(1), 2017, pp.59-87

\title{
COMPARISON OF POWER OPERATIONS IN MORAVA E-THEORIES
}

\author{
TAKESHI TORII
}

(communicated by Donald M. Davis)

\begin{abstract}
There is a Hopf algebroid without antipode which is the dual of the algebra of power operations in Morava E-theory. In this paper we compare the category of comodules over the Hopf algebroid in the $n$th Morava $E$-theory with that in the $(n+1)$ st Morava $E$-theory. We show that the $n$th Morava $E$-theory of a finite complex with power operations can be obtained from the $(n+1)$ st Morava $E$-theory with power operations.
\end{abstract}

\section{Introduction}

An $H_{\infty}$-ring spectrum gives rise to power operations in the corresponding cohomology theory. Steenrod operations in $\bmod p$ cohomology and Adams operations in $K$-theory are examples of power operations associated to $H_{\infty}$-ring spectrum structures. Roughly speaking, giving an $H_{\infty}$-ring spectrum structure on a spectrum is equivalent to giving power operations in the corresponding cohomology theory. Thus, it is important to understand the structure of power operations for an $H_{\infty}$-ring spectrum.

Let $E_{n}$ be the $n$th Morava $E$-theory spectrum at a prime $p$. Recall that $E_{n}$ is an even periodic commutative ring spectrum and the degree 0 coefficient ring $E_{n}^{0}=$ $\pi_{0}\left(E_{n}\right)$ is

$$
W\left(\mathbb{F}_{p^{n}}\right) \llbracket w_{1}, \ldots, w_{n-1} \rrbracket,
$$

where $W\left(\mathbb{F}_{p^{n}}\right)$ is the ring of Witt vectors with coefficients in the finite field $\mathbb{F}_{p^{n}}$ with $p^{n}$ elements. Power operations in Morava $E$-theory were first studied by Ando in $[\mathbf{1}, \mathbf{2}]$, where he used power operations in complex cobordism to produce power operations in Morava E-theory. In [6] Goerss-Hopkins showed that Morava E-theory supports an $E_{\infty}$-ring spectrum structure unique up to homotopy, which implies the existence of an $H_{\infty}$-ring spectrum structure on Morava $E$-theory. Power operations are related to the cohomology groups of classifying spaces of symmetric groups and these structures in Morava $E$-theory are related to level structures and finite subgroups on the associated formal group. The moduli spaces of level structures and finite subgroups on formal groups and the Morava $E$-theory of classifying spaces of symmetric groups

This work was partially supported by JSPS KAKENHI Grant Number 25400092.

Received January 12, 2015, revised October 23, 2015, June 11, 2016; published on March 22, 2017. 2010 Mathematics Subject Classification: 55N22, 14L05, 55S25.

Key words and phrases: Morava E-theory, power operation, $p$-divisible group, Hopf algebroid.

Article available at http://dx.doi.org/10.4310/HHA.2017.v19.n1.a4

Copyright (C) 2017, International Press. Permission to copy for private use granted. 
were studied by Strickland in $[\mathbf{1 3}, \mathbf{1 4}]$. More generally, Ando-Hopkins-Strickland in [3] studied the algebraic geometry of complex orientable $H_{\infty}$-ring spectra. Based on these works, the algebra of power operations in Morava $E$-theory was introduced by Rezk in $[\mathbf{1 2}]$.

The stable homotopy category is considered to be built from $K(n)$-local categories, where $K(n)$ is the $n$th Morava $K$-theory. Thus, it is important to understand the relationship between $K(n)$-local categories. In $[\mathbf{1 5}, \mathbf{1 6}]$ for this purpose we introduced the generalized Chern character

$$
\text { ch: } E_{n+1} \rightarrow \mathbb{B},
$$

where $E_{n+1}$ is the $(n+1)$ st Morava $E$-theory spectrum and $\mathbb{B}$ is a $K(n)$-local commutative ring spectrum that is an extension of $E_{n}$. We define $\mathbb{B}_{n}$ to be the $K(n)$ localization of $E_{n} \wedge E_{n+1}$ :

$$
\mathbb{B}_{n}=L_{K(n)}\left(E_{n} \wedge E_{n+1}\right) .
$$

In this paper we assume that the residue field of the local ring $\pi_{0}(\mathbb{B})$ is the composite field of $\mathbb{F}_{p^{n}}$ and $\mathbb{F}_{p^{n+1}}$. In this case the commutative ring spectrum $\mathbb{B}$ is equivalent to $\mathbb{B}_{n}$ (see Lemma 4.1 below).

In [19] we discussed the relationship between the Hecke operators in the $n$th Morava $E$-theory and those in the $(n+1)$ st Morava $E$-theory via the $\mathbb{B}_{n}$-theory. Hecke operators in Morava $E$-theory are defined by averaging power operations. In this paper we compare the category of modules over the algebra of power operations in the $n$th Morava $E$-theory with that in the $(n+1)$ st Morava $E$-theory. Actually, we compare the categories of comodules over the dual Hopf algebroids in this paper. There is a Hopf algebroid without antipode which is the dual of the algebra of power operations, and the category of modules over the algebra of power operations is equivalent to the category of comodules over the dual Hopf algebroid.

Since $E_{n}$ is even periodic, we have the associated degree 0 one dimensional formal Lie group $\mathbf{F}_{n}$ over $E_{n}^{0}$. The formal group $\mathbf{F}_{n}$ is a universal deformation of the Honda formal group $\overline{\mathbf{F}}_{n}$ over $\mathbb{F}_{p^{n}}$. Let $\mathbb{S}_{n}$ be the $n$th Morava stabilizer group, which is the automorphism group of $\overline{\mathbf{F}}_{n}$ over $\mathbb{F}_{p^{n}}$ and let $\operatorname{Gal}\left(\mathbb{F}_{p^{n}} / \mathbb{F}_{p}\right)$ be the Galois group of $\mathbb{F}_{p^{n}}$ over the prime field $\mathbb{F}_{p}$. The group $\operatorname{Gal}\left(\mathbb{F}_{p^{n}} / \mathbb{F}_{p}\right)$ acts on $\mathbb{S}_{n}$ and the $n$th extended Morava stabilizer group $\mathbb{G}_{n}$ is defined to be the semidirect product

$$
\operatorname{Gal}\left(\mathbb{F}_{p^{n}} / \mathbb{F}_{p}\right) \ltimes \mathbb{S}_{n} .
$$

We can identify $\mathbb{G}_{n}$ with the group of multiplicative automorphisms of $E_{n}$ in the stable homotopy category. In particular, $\mathbb{G}_{n}$ acts naturally on the $E_{n}$-cohomology ring $E_{n}^{0}(X)$ for any space $X$.

In [16] we essentially showed that the $E_{n}$-cohomology of a finite complex $X$ with action of $\mathbb{G}_{n}$ can be obtained from the $E_{n+1}$-cohomology of $X$ with action of $\mathbb{G}_{n+1}$. This means that there is a functor

$$
C: \mathbb{G}_{n+1}-\operatorname{Mod}\left(E_{n+1}^{0}\right) \longrightarrow \mathbb{G}_{n}-\operatorname{Mod}\left(E_{n}^{0}\right),
$$

where $\mathbb{G}_{n}-\operatorname{Mod}\left(E_{n}^{0}\right)$ is the category of twisted $E_{n}^{0}-\mathbb{G}_{n}$-modules, and there is a natural isomorphism $E_{n}^{0}(X) \cong C\left(E_{n+1}^{0}(X)\right)$ of commutative twisted $E_{n}^{0}-\mathbb{G}_{n}$-algebras for any finite complex $X$. The functor $C$ is given by $C(M)=\left(\mathbb{B}_{n}^{0} \otimes_{E_{n+1}^{0}} M\right)^{\mathbb{G}_{n+1}}$ for $M \in$ $\mathbb{G}_{n+1}-\operatorname{Mod}\left(E_{n+1}^{0}\right)$, where $\mathbb{G}_{n+1}$ acts on $\mathbb{B}_{n}^{0} \otimes_{E_{n+1}^{0}} M$ diagonally. 
Let $\mathcal{S}_{n}$ be the Hopf algebroid without antipode which is the dual of the algebra of power operations in the $n$th Morava $E$-theory. We denote by $\mathbb{G}_{n}$ - $\operatorname{Comod}\left(\mathcal{S}_{n}\right)$ the category of comodules over $\mathcal{S}_{n}$ with compatible $\mathbb{G}_{n}$-action, and by $\mathbb{G}_{n}$-ComodAlg $\left(\mathcal{S}_{n}\right)$ the category of commutative $\mathcal{S}_{n}$-comodule algebras with compatible $\mathbb{G}_{n}$-action. The following is our first main theorem.

Theorem 1.1 (Theorem 5.14). There is a functor

$$
C: \mathbb{G}_{n+1}-\operatorname{Comod}\left(\mathcal{S}_{n+1}\right) \longrightarrow \mathbb{G}_{n}-\operatorname{Comod}\left(\mathcal{S}_{n}\right),
$$

which is given by

$$
C(M)=\left(\mathbb{B}_{n}^{0} \otimes_{E_{n+1}^{0}} M\right)^{\mathbb{G}_{n+1}},
$$

for $M \in \mathbb{G}_{n+1}$-Comod $\left(\mathcal{S}_{n+1}\right)$. Furthermore, this functor induces a functor

$$
C: \mathbb{G}_{n+1} \text {-ComodAlg }\left(\mathcal{S}_{n+1}\right) \longrightarrow \mathbb{G}_{n} \text { - } \operatorname{ComodAlg}\left(\mathcal{S}_{n}\right) \text {. }
$$

Combining this theorem with the result in $[\mathbf{1 6}]$, we obtain the following theorem, which is our second main theorem.

Theorem 1.2 (Theorem 5.17). For any finite complex $X$, there is a natural isomorphism

$$
E_{n}^{0}(X) \cong C\left(E_{n+1}^{0}(X)\right)
$$

of commutative $\mathcal{S}_{n}$-comodule algebras with compatible $\mathbb{G}_{n}$-action.

The organization of this paper is as follows: In $\S 2$, we fix notation used throughout this paper. In $\S 3$, we review the Hopf algebroid without antipode which is the dual of the algebra of power operations in Morava $E$-theory. We also study the action of the extended Morava stabilizer group on a comodule over the Hopf algebroid. In $\S 4$, we construct a Hopf algebroid $\mathcal{S}_{\mathbb{B}}$ over $\mathbb{B}_{n}^{0}$, which is the base change of $\mathcal{S}_{n+1}$ along the map ch: $E_{n+1}^{0} \rightarrow \mathbb{B}_{n}^{0}$. For this purpose we study the moduli space of finite subgroups of the $p$-divisible group over $\mathbb{B}_{n}^{0}$ obtained from the $p$-divisible group associated to the formal Lie group of $E_{n+1}$ by base change along ch. We show that there is an adjoint pair of functors between the category of comodules over $\mathcal{S}_{\mathbb{B}}$ and the category of comodules over $\mathcal{S}_{n+1}$. In $\S 5$, we study the $\mathbb{G}_{n} \times \mathbb{G}_{n+1}$-action on the Hopf algebroid $\mathcal{S}_{\mathbb{B}}$ and the category of comodules over $\mathcal{S}_{\mathbb{B}}$ with compatible $\mathbb{G}_{n} \times \mathbb{G}_{n+1}$-action. We show that the adjoint pair of functors constructed in $\S 4$ upgrades to an adjoint pair of functors between the category of comodules over $\mathcal{S}_{\mathbb{B}}$ with compatible $\mathbb{G}_{n} \times \mathbb{G}_{n+1}$-action and the category of comodules over $\mathcal{S}_{n+1}$ with compatible $\mathbb{G}_{n+1}$-action. Furthermore, we construct a quotient Hopf algebroid $\mathcal{S}_{\mathbb{B}}^{0}$ of $\mathcal{S}_{\mathbb{B}}$. We show that $\mathcal{S}_{\mathbb{B}}^{0}$ is obtained from $\mathcal{S}_{n}$ by base change along a map $E_{n}^{0} \rightarrow \mathbb{B}_{n}^{0}$ and that there is an adjoint pair of functors between the category of comodules over $\mathcal{S}_{n}$ with compatible $\mathbb{G}_{n}$-action and the category of comodules over $\mathcal{S}_{\mathbb{B}}^{0}$ with compatible $\mathbb{G}_{n} \times \mathbb{G}_{n+1}$-action. Using these results, we construct the functor $C$ from the category of $\mathcal{S}_{n+1}$-comodules with compatible $\mathbb{G}_{n+1}$-action to the category of $\mathcal{S}_{n}$-comodules with compatible $\mathbb{G}_{n}$-action, and prove our main theorems. 


\section{Notation}

We fix a prime number $p$ and a positive integer $n$. Let $K(n)$ be the $n$th Morava $K$-theory at $p$, and let $E_{n}$ be the $n$th Morava $E$-theory at $p$.

Let $\mathcal{C} \mathcal{L}$ be the category of complete Noetherian local rings with residue field of characteristic $p$ and local ring homomorphisms. For $R \in \mathcal{C} \mathcal{L}$ we denote by $\mathcal{C} \mathcal{L}_{R}$ the under category $(R \downarrow \mathcal{C L})$ whose objects are maps in $\mathcal{C} \mathcal{L}$ with source $R$ and whose morphisms are commutative triangles in $\mathcal{C} \mathcal{L}$. We denote by $\operatorname{Spf}(R)$ for $R \in \mathcal{C} \mathcal{L}$ the functor from $\mathcal{C} \mathcal{L}$ to the category of sets given by

$$
\operatorname{Spf}(R)(S)=\operatorname{Hom}_{\mathcal{C} \mathcal{L}}(R, S) .
$$

For a complete Noetherian semilocal ring $R$ with residue fields of characteristic $p$, we set

$$
\operatorname{Spf}(R)=\coprod_{i=1}^{n} \operatorname{Spf}\left(R_{i}\right),
$$

where $R=\prod_{i=1}^{n} R_{i}$ is the decomposition of $R$ into a finite product of local rings in $\mathcal{C L}$.

For a commutative ring $E$, we denote by $\operatorname{Mod}(E)$ the category of $E$-modules and $E$ module homomorphisms. We let $\mathrm{CAlg}(E)$ be the category of commutative $E$-algebras and $E$-algebra homomorphisms.

\section{Power operations in Morava E-theory}

In [6] Goerss-Hopkins showed that Morava $E$-theory supports an $E_{\infty}$-ring spectrum structure unique up to homotopy. Thus, we can consider power operations in the $E_{n}$-cohomology of spaces. In [12] Rezk introduced the algebra of power operations in Morava $E$-theory, which naturally acts on the degree 0 homotopy group of any $K(n)$-local commutative $E_{n}$-algebra. In this section, we review the algebra of power operations in Morava E-theory.

\subsection{Finite subgroups of a formal Lie group}

Let $\mathbf{X}$ be a one dimensional formal Lie group over $R \in \mathcal{C} \mathcal{L}$. We assume that $\mathbf{X}$ has finite height. For a map $f: R \rightarrow S$ in $\mathcal{C} \mathcal{L}$, we denote by $f^{*} \mathbf{X}$ or $\mathbf{X}_{S}$ the base change of $\mathbf{X}$ along $f$.

A divisor on $\mathbf{X}$ is a closed subscheme which is finite and flat over $R$. The degree of a divisor is the rank of the coordinate ring over $R$. A finite subgroup of a one dimensional formal Lie group was defined by Strickland in $[\mathbf{1 3}, \S 5]$.

Definition $3.1([13, \S 5])$. A finite subgroup of $\mathbf{X}$ is a divisor which is also a subgroup scheme.

Note that the degree of a finite subgroup is a power of $p$ (see, for example, $[\mathbf{1 3}$, Proposition 17]). For a nonnegative integer $m$, we define the functor

$$
\operatorname{Sub}(\mathbf{X}, m)
$$

over $\operatorname{Spf}(R)$ by assigning to $U \in \mathcal{C} \mathcal{L}_{R}$ the set of all the finite subgroups of $\mathbf{X}_{U}$ of degree $p^{m}$. By [13, Theorem 42], the functor $\operatorname{Sub}(\mathbf{X}, m)$ is representable by a complete 
Noetherian local $E_{n}^{0}$-algebra. We denote by $S\left(\mathbf{F}_{n}, m\right)$ the representing ring so that

$$
\operatorname{Sub}\left(\mathbf{F}_{n}, m\right)=\operatorname{Spf}\left(S\left(\mathbf{F}_{n}, m\right)\right) .
$$

Note that $S\left(\mathbf{F}_{n}, m\right)$ is a finitely generated free $E_{n}^{0}$-module (see [13, Theorem 42]).

Let $\mathbf{Y}$ be a one dimensional formal Lie group over $S \in \mathcal{C} \mathcal{L}$. We suppose there is a map $f: R \rightarrow S$ in $\mathcal{C L}$ which induces an isomorphism $\mathbf{Y} \cong f^{*} \mathbf{X}$. A finite subgroup of $\mathbf{Y}$ can be regarded as a finite subgroup of $f^{*} \mathbf{X}=\mathbf{X}_{S}$. This implies that there is an isomorphism of functors

$$
\operatorname{Sub}(\mathbf{Y}, m) \stackrel{\cong}{\longrightarrow} \operatorname{Sub}(\mathbf{X}, m) \times_{\operatorname{Spf}(R)} \operatorname{Spf}(S) .
$$

For a finite subgroup $K$ of $\mathbf{X}$ with degree $p^{m}$, we can construct the quotient formal group $\mathbf{X} / K$ over $R$ (see [13, Theorem 19]). The formal group $\mathbf{X} / K$ sits in an exact sequence

$$
0 \longrightarrow K \longrightarrow \mathbf{X} \longrightarrow \mathbf{X} / K \longrightarrow 0,
$$

of fppf sheaves of abelian groups on $\operatorname{Spf}(R)$. Let $k$ be the residue field of $R$ and let $\pi: R \rightarrow k$ be the reduction map. Since $\pi^{*} \mathbf{X}$ is a formal group over a field of characteristic $p$, we see that $\pi^{*}(\mathbf{X} / K)$ is isomorphic to $\left(\sigma^{m}\right)^{*} \pi^{*} \mathbf{X}$, where $\sigma$ is the absolute Frobenius for $k$, and that the quotient map $\pi^{*} \mathbf{X} \rightarrow \pi^{*}(\mathbf{X} / K)$ can be identified with the $m$ th iterate of the relative Frobenius.

\subsection{The graded Hopf algebroid $\mathcal{S}_{n}$ without antipode}

First, we recall graded Hopf algebroids without antipode (see $[\mathbf{1 2}, \S 5.5]$ ).

Let $E$ be a commutative ring and let $\{S(m)\}$ be a family of commutative rings indexed by nonnegative integers $m$. We suppose there are ring homomorphisms

$$
\begin{aligned}
s_{m}: & E \longrightarrow S(m), \\
t_{m}: & E \longrightarrow S(m), \\
i: & S(0) \longrightarrow E, \\
c_{m^{\prime}, m}: & S\left(m+m^{\prime}\right) \longrightarrow S\left(m^{\prime}\right) \underset{s, E, t}{\otimes} S(m),
\end{aligned}
$$

for all $m, m^{\prime} \geqslant 0$. In the tensor product $S\left(m^{\prime}\right) \underset{s, E, t}{\otimes} S(m)$ we regard $S\left(m^{\prime}\right)$ as an $E$ module via $s_{m^{\prime}}$ and $S(m)$ via $t_{m}$. We use abbreviations $s, t, c$ for $s_{m}, t_{m}, c_{m^{\prime}, m}$ for $m, m^{\prime} \geqslant 0$, respectively, when there is no confusion. The data $\Gamma=(E,\{S(m)\}, s, t, i, c)$ is said to be a graded Hopf algebroid without antipode if it represents a graded affine category scheme, that is, if it corepresents a functor from the category of commutative rings to the category of graded categories (see $[\mathbf{1 2}, \S 5.5]$ for the definitions of a graded category and a graded affine category scheme), where $E$ and $S(m)$ corepresent the set of objects and the set of morphisms of degree $m$ of a graded category, respectively, and the maps $s, t, i, c$ correspond to source, target, identity, and composition, respectively. An $E$-module $M$ is said to be a (left) $\Gamma$-comodule if $M$ is equipped with E-module homomorphisms

$$
\psi_{M, m}: M \longrightarrow S(m) \underset{s, E}{\otimes} M,
$$


for all $m \geqslant 0$, which satisfy the coassociativity and counit conditions. Note that the $E$ module structure on $S(m) \otimes_{s, E} M$ is given by the $E$-module structure on $S(m)$ via $t_{m}$. An $E$-module map $f: M \rightarrow N$ between $\Gamma$-comodules is said to be a $\Gamma$-comodule map if $f$ respects the comodule structure maps, that is, if the identity $\left(i d_{S(m)} \otimes f\right) \circ \psi_{M, m}=$ $\psi_{N, m} \circ f$ holds, for all $m \geqslant 0$. We denote by

\section{$\operatorname{Comod}(\Gamma)$}

the category of $\Gamma$-comodules and $\Gamma$-comodule maps.

The category $\operatorname{Comod}(\Gamma)$ of $\Gamma$-comodules is a symmetric monoidal category with the tensor product $\otimes_{E}$ and the unit object $E$. A $\Gamma$-comodule $M$ is said to be a commutative $\Gamma$-comodule algebra if $M$ is a commutative monoid object in $\operatorname{Comod}(\Gamma)$. A $\Gamma$-comodule map $f: M \rightarrow N$ between commutative $\Gamma$-comodule algebras is said to be a commutative $\Gamma$-comodule algebra map if $f$ is a map of commutative monoid objects in $\operatorname{Comod}(\Gamma)$. We denote by

\section{ComodAlg $(\Gamma)$}

the category of commutative $\Gamma$-comodule algebras and commutative $\Gamma$-comodule algebra maps (see, for example, [11, Appendix A1.1] for the definition of a comodule algebra over a Hopf algebroid).

If $S(m)$ is a finitely generated projective $E$-module for all $m \geqslant 0$, then there is a graded bialgebra $\mathcal{B}$ over $E$ (see $[\mathbf{1 2}, \S \S 5.2$ and 5.5] for the definition of a graded bialgebra) and the category $\operatorname{Comod}(\Gamma)$ of $\Gamma$-comodules is equivalent to the category of modules over the graded bialgebra $\mathcal{B}$ by [12, Proposition 5.3]. Hence in this case the category ComodAlg $(\Gamma)$ of commutative $\Gamma$-comodule algebras is equivalent to the category of $\mathcal{B}$-algebras (see $[\mathbf{1 2}, \S 5.4]$ for the definition of an algebra over a bialgebra).

Recall that $\mathbf{F}_{n}$ over $E_{n}^{0}$ is a universal deformation of the Honda formal group $\overline{\mathbf{F}}_{n}$. If $\mathbf{X}$ is a deformation of $\overline{\mathbf{F}}_{n}$ to $R \in \mathcal{C} \mathcal{L}$ and $A$ is a finite subgroup of $\mathbf{X}$ with degree $p^{m}$, then $\mathbf{X} / A$ is also a deformation of $\overline{\mathbf{F}}_{n}$, and hence there is a unique map $E_{n}^{0} \rightarrow R$ in $\mathcal{C} \mathcal{L}$ which classifies $\mathbf{X} / A$. This implies that there is a map

$$
t_{m}: E_{n}^{0} \longrightarrow S\left(\mathbf{F}_{n}, m\right) \text {. }
$$

We let

$$
s_{m}: E_{n}^{0} \longrightarrow S\left(\mathbf{F}_{n}, m\right)
$$

be the inclusion, and we let

$$
i: S\left(\mathbf{F}_{n}, 0\right) \longrightarrow E_{n}^{0}
$$

be the canonical isomorphism. Suppose $\mathbf{X}^{\prime}$ is a deformation of $\overline{\mathbf{F}}_{n}$ to $R \in \mathcal{C} \mathcal{L}$ such that $\mathbf{X} / A$ is $*$-isomorphic to $\mathbf{X}^{\prime}$. For a finite subgroup $A^{\prime}$ of $\mathbf{X}^{\prime}$ with degree $p^{m^{\prime}}$, the kernel of the composition

$$
\mathbf{X} \longrightarrow \mathbf{X} / A \stackrel{\cong}{\longrightarrow} \mathbf{X}^{\prime} \longrightarrow \mathbf{X}^{\prime} / A^{\prime}
$$

is a finite subgroup of $\mathbf{X}$ of degree $p^{m+m^{\prime}}$. This implies that there is a map

$$
c_{m^{\prime}, m}: S\left(\mathbf{F}_{n}, m+m^{\prime}\right) \longrightarrow S\left(\mathbf{F}_{n}, m^{\prime}\right) \underset{s, E_{n}^{0}, t}{\otimes} S\left(\mathbf{F}_{n}, m\right),
$$

in $\mathcal{C} \mathcal{L}$. By $[\mathbf{1 2}, \S 11.6]$, the data $\mathcal{S}_{n}=\left(E_{n}^{0},\left\{S\left(\mathbf{F}_{n}, m\right)\right\}, s, t, i, c\right)$ represents a formal graded category scheme, that is, $\mathcal{S}_{n}$ is a formal graded Hopf algebroid without 
antipode. Thus, we can consider the category $\operatorname{Comod}\left(\mathcal{S}_{n}\right)$ of $\mathcal{S}_{n}$-comodules and the category Comod $\operatorname{Alg}\left(\mathcal{S}_{n}\right)$ of commutative $\mathcal{S}_{n}$-comodule algebras.

\subsection{The action of $\mathbb{G}_{n}$ on $\mathcal{S}_{n}$}

Recall that $\mathbb{G}_{n}$ is the $n$th extended Morava stabilizer group, which is the semidirect product of the automorphism group $\mathbb{S}_{n}$ of the Honda formal group $\overline{\mathbf{F}}_{n}$ over $\mathbb{F}_{p^{n}}$ and the Galois group $\operatorname{Gal}\left(\mathbb{F}_{p^{n}} / \mathbb{F}_{p}\right)$ of $\mathbb{F}_{p^{n}}$ over the prime field $\mathbb{F}_{p}$. The action of $\mathbb{G}_{n}$ on $\overline{\mathbf{F}}_{n}$ over $\operatorname{Spec}\left(\mathbb{F}_{p^{n}}\right)$ extends to an action on $\mathbf{F}_{n}$ over $\operatorname{Spf}\left(E_{n}^{0}\right)$. Hence we obtain a commutative diagram of functors

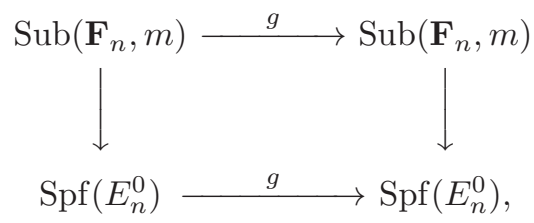

for any $g \in \mathbb{G}_{n}$ and any $m \geqslant 0$. This gives rise to an action of $\mathbb{G}_{n}$ on the representing ring $S\left(\mathbf{F}_{n}, m\right)$, which is compatible with the action on $E_{n}^{0}$. Using the universality of the ring $S\left(\mathbf{F}_{n}, m\right)$, we obtain the following proposition.

Proposition 3.2. The structure maps $s, t, i, c$ of $\mathcal{S}_{n}$ are $\mathbb{G}_{n}$-equivariant and $\mathbb{G}_{n}$ acts on the graded Hopf algebroid $\mathcal{S}_{n}$ without antipode.

\subsection{Power operations in $K(n)$-local commutative $E_{n}$-algebras}

For a space $X$, we denote by $\Sigma_{+}^{\infty} X$ the suspension spectrum of $X$ with a disjoint base point. By [6], Morava $E$-theory $E_{n}$ is an $E_{\infty}$-ring spectrum unique up to homotopy. Thus, we can consider the category of $K(n)$-local commutative $E_{n}$-algebras. For a $K(n)$-local commutative $E_{n}$-algebra $R$, we have a structure map

$$
\nu_{k}: \Sigma_{+}^{\infty} E \Sigma_{k} \underset{\Sigma_{k}}{\wedge} R^{\wedge k} \longrightarrow R
$$

for each nonnegative integer $k$, where $\Sigma_{k}$ is the symmetric group of degree $k, E \Sigma_{k}$ is its universal space, and $R^{\wedge k}$ is the $k$-fold smash power of $R$. Let $S^{0}$ be the sphere spectrum and let $\left(S^{0}\right)^{\wedge k}$ be the $k$-fold smash power of $S^{0}$. For a map $f: S^{0} \longrightarrow R$, by the composition

$$
\Sigma_{+}^{\infty} B \Sigma_{k} \cong \Sigma_{+}^{\infty} E \Sigma_{k} \underset{\Sigma_{k}}{\wedge}\left(S^{0}\right)^{\wedge k} \stackrel{\mathrm{id} \wedge f^{\wedge k}}{\longrightarrow} \Sigma_{+}^{\infty} E \Sigma_{k} \underset{\Sigma_{k}}{\wedge} R^{\wedge k} \stackrel{\nu_{k}}{\longrightarrow} R
$$

we obtain a natural map

$$
\mathrm{P}_{k}: \pi_{0}(R) \longrightarrow R^{0}\left(B \Sigma_{k}\right)
$$

where $B \Sigma_{k}$ is the classifying space of $\Sigma_{k}$. Note that $\mathrm{P}_{k}$ is not an additive map in general.

For any $m \geqslant 0$ and $0 \leqslant i \leqslant p^{m}$, we have the transfer map $E_{n}^{0}\left(B\left(\Sigma_{i} \times \Sigma_{p^{m}-i}\right)\right) \rightarrow$ $E_{n}^{0}\left(B \Sigma_{p^{m}}\right)$ for the subgroup $\Sigma_{i} \times \Sigma_{p^{m}-i}$ of $\Sigma_{p^{m}}$. Let $J$ be the ideal of $E_{n}^{0}\left(B \Sigma_{p^{m}}\right)$ generated by the images of the transfer maps for $0<i<p^{m}$. By [14, Theorem 9.2], there is an isomorphism of rings

$$
S\left(\mathbf{F}_{n}, m\right) \cong E_{n}^{0}\left(B \Sigma_{p^{m}}\right) / J .
$$

We denote by $\pi_{m}$ the quotient map $E_{n}^{0}\left(B \Sigma_{p^{m}}\right) \rightarrow S\left(\mathbf{F}_{n}, m\right)$. Recall that $E_{n}^{0}\left(B \Sigma_{k}\right)$ 
is a finitely generated free $E_{n}^{0}$-module for any $k$ (see, for example, [13, Proposition 3.6]). This implies that there is an isomorphism

$$
R^{0}\left(B \Sigma_{k}\right) \cong E_{n}^{0}\left(B \Sigma_{k}\right) \otimes_{E_{n}^{0}} \pi_{0}(R)
$$

The composition

$$
\psi_{m}^{R}: \pi_{0}(R) \stackrel{\mathrm{P}_{p^{m}}}{\longrightarrow} R^{0}\left(B \Sigma_{p^{m}}\right) \cong E_{n}^{0}\left(B \Sigma_{p^{m}}\right) \underset{E_{n}^{0}}{\otimes} \pi_{0}(R) \stackrel{\pi_{m} \otimes \mathrm{id}}{\longrightarrow} S\left(\mathbf{F}_{n}, m\right) \underset{s, E_{n}^{0}}{\otimes} \pi_{0}(R)
$$

gives rise to an $\mathcal{S}_{n}$-comodule structure on $\pi_{0}(R)$, and hence $\pi_{0}(R)$ is a commutative $\mathcal{S}_{n}$-comodule algebra for any $K(n)$-local commutative $E_{n}$-algebra $R$. In particular, when $R=E_{n}$, we obtain the map

$$
t_{m}=\psi_{m}^{E_{n}}: E_{n}^{0} \longrightarrow S\left(\mathbf{F}_{n}, m\right) .
$$

For a space $X$, we denote by $E_{n}^{X}$ the function spectrum. Note that $E_{n}^{X}$ is a $K(n)$ local commutative $E_{n}$-algebra for any space $X$. When $R=E_{n}^{X}$, we obtain a natural operation

$$
\psi_{m}: E_{n}^{0}(X) \longrightarrow S\left(\mathbf{F}_{n}, m\right) \otimes_{s, E_{n}^{0}} E_{n}^{0}(X) .
$$

In particular, when $X$ is the infinite complex projective space $\mathbb{C P}^{\infty}$, we obtain a map $\psi_{m}: E_{n}^{0}\left(\mathbb{C P}^{\infty}\right) \rightarrow S\left(\mathbf{F}_{n}, m\right) \otimes_{s, E_{n}^{0}} E_{n}^{0}\left(\mathbb{C P}^{\infty}\right)$. This induces an isogeny of formal groups

$$
\Psi_{m}^{E_{n}}: s_{m}^{*} \mathbf{F}_{n} \longrightarrow t_{m}^{*} \mathbf{F}_{n}
$$

whose kernel is a universal finite subgroup of $s_{m}^{*} \mathbf{F}_{n}$ over $S\left(\mathbf{F}_{n}, m\right)$.

Now we regard $S\left(\mathbf{F}_{n}, m\right)$ as an $E_{n}^{0}$-module via the inclusion $s_{m}$ for any $m \geqslant 0$. We define $\mathrm{PW}_{n}(m)$ to be the abelian group of all the $E_{n}^{0}$-module homomorphisms from $S\left(\mathbf{F}_{n}, m\right)$ to $E_{n}^{0}$, and we set

$$
\mathrm{PW}_{n}=\bigoplus_{m \geqslant 0} \mathrm{PW}_{n}(m) .
$$

We can regard $\mathrm{PW}_{n}$ as a graded module, and $\mathrm{PW}_{n}$ supports the structure of a graded twisted cocommutative $E_{n}^{0}$-bialgebra. We call $\mathrm{PW}_{n}$ the algebra of power operations in Morava $E$-theory (see $[\mathbf{1 2}, \S 5.2]$ ).

For $\gamma \in \mathrm{PW}_{n}(m)$, the composition

$$
\pi_{0}(R) \stackrel{\psi_{m}^{R}}{\longrightarrow} S\left(\mathbf{F}_{n}, m\right) \underset{s, E_{n}^{0}}{\otimes} \pi_{0}(R) \stackrel{\gamma \otimes \mathrm{id}}{\longrightarrow} \pi_{0}(R)
$$

gives rise to a right action of $\mathrm{PW}_{n}$ on $\pi_{0}(R)$, and hence $\pi_{0}(R)$ is a $\mathrm{PW}_{n}$-module. Furthermore, $\pi_{0}(R)$ is a commutative $\mathrm{PW}_{n}$-module algebra. By [12, Proposition 5.3], the category of $\mathrm{PW}_{n}$-modules is equivalent to the category of $\mathcal{S}_{n}$-comodules $\operatorname{Comod}\left(\mathcal{S}_{n}\right)$, and the category of commutative $\mathrm{PW}_{n}$-module algebras is equivalent to the category of commutative $\mathcal{S}_{n}$-comodule algebras $\operatorname{ComodAlg}\left(\mathcal{S}_{n}\right)$.

\section{5. $\mathcal{S}_{n}$-comodules with compatible $\mathbb{G}_{n}$-action}

Let $E$ be a commutative ring. We suppose that a group $G$ acts on $E$. An $E$-module $M$ is said to be a twisted $E-G$-module if $M$ is equipped with a $G$-action such that

$$
g(e m)=g(e) g(m)
$$


for any $g \in G, e \in E$, and $m \in M$. We denote by

$$
G-\operatorname{Mod}(E)
$$

the category of twisted $E$ - $G$-modules and $G$-equivariant $E$-module homomorphisms. This category is an immediate abstraction of the category of continuous Galois equivariant twisted $S_{n}-E_{n *}^{\wedge}$-modules studied by Devinatz in [4]. Though we are not aware of a reference for the following concepts, they are all natural, given the context that we are working in. The category $G-\operatorname{Mod}(E)$ is a symmetric monoidal category with the tensor product $\otimes_{E}$ and the unit object $E$. A twisted $E-G$-module is said to be a twisted commutative $E$ - $G$-algebra if it is a commutative monoid object in $G$-Mod $(E)$. A map of twisted $E$ - $G$-modules between twisted commutative $E$ - $G$-algebras is said to be a twisted commutative $E$ - $G$-algebra map if it is a map of commutative monoids in $G-\operatorname{Mod}(E)$. We denote by

$$
G-\mathrm{CAlg}(E)
$$

the category of twisted commutative $E$ - $G$-algebras and twisted commutative $E$ - $G$ algebra maps.

Let $\Gamma=(E,\{S(m)\}, s, t, i, c)$ be a graded Hopf algebroid without antipode. We suppose that $G$ acts on $\Gamma$. A $\Gamma$-comodule $M$ is said to be a $\Gamma$-comodule with compatible $G$-action if $M$ is a twisted $E$ - $G$-module such that the comodule structure map $M \rightarrow$ $S(m) \otimes_{s, E} M$ is $G$-equivariant for any $m \geqslant 0$, where $G$ acts diagonally on the righthand side. We denote by

$$
G-\operatorname{Comod}(\Gamma)
$$

the category of $\Gamma$-comodules with compatible $G$-action and $G$-equivariant comodule maps.

It is easy to see that $G$ - $\operatorname{Comod}(\Gamma)$ is a symmetric monoidal category. We say that a commutative monoid object in $G$-Comod $(\Gamma)$ is a commutative $\Gamma$-comodule algebra with compatible $G$-action. We denote by

$$
G-\operatorname{ComodAlg}(\Gamma)
$$

the category of commutative $\Gamma$-comodule algebras with compatible $G$-action and $G$ equivariant comodule algebra maps.

By Proposition 3.2, the extended Morava stabilizer group $\mathbb{G}_{n}$ acts on the graded Hopf algebroid $\mathcal{S}_{n}$ without antipode. Hence we can consider $\mathbb{G}_{n}$ - $\operatorname{Comod}\left(\mathcal{S}_{n}\right)$ the category of $\mathcal{S}_{n}$-comodules with compatible $\mathbb{G}_{n}$-action. The group $\mathbb{G}_{n}$ acts on $\mathrm{PW}{ }_{n}$ by

$$
(g \gamma)(x)=g\left(\gamma\left(g^{-1} x\right)\right),
$$

for $\gamma \in \mathrm{PW}_{n}(m), x \in S\left(\mathbf{F}_{n}, m\right)$ and $g \in \mathbb{G}_{n}$. A PW $n^{-m o d u l e ~} M$ is said to be a $\mathrm{PW}_{n^{-}}$ module with compatible $\mathbb{G}_{n}$-action if $M$ is a twisted $E_{n}^{0}$ - $\mathbb{G}_{n}$-module and the action map $M \times \mathrm{PW}_{n} \rightarrow M$ is $\mathbb{G}_{n}$-equivariant, where $\mathbb{G}_{n}$ acts diagonally on the left-hand side. The following proposition is easily obtained.

Proposition 3.3. The category of $\mathrm{PW}_{n}$-modules with compatible $\mathbb{G}_{n}$-action and $\mathbb{G}_{n}$ equivariant $E_{n}^{0}$-module homomorphisms is equivalent to the category of $\mathcal{S}_{n}$-comodules with compatible $\mathbb{G}_{n}$-action $\mathbb{G}_{n}-\operatorname{Comod}\left(\mathcal{S}_{n}\right)$. This equivalence restricts to an equivalence between the category of commutative $\mathrm{PW}_{n}$-module algebras with compatible 
$\mathbb{G}_{n}$-action and the category of commutative $\mathcal{S}_{n}$-comodule algebras with compatible $\mathbb{G}_{n}$-action $\mathbb{G}_{n}$-ComodAlg $\left(\mathcal{S}_{n}\right)$.

For the remainder of this paper we use comodules over the Hopf algebroid $\mathcal{S}_{n}$ without antipode instead of modules over the algebra $\mathrm{PW}_{n}$ of power operations.

\section{Operations in $\mathbb{B}_{n}$-theory}

For a $p$-divisible group $\mathbf{X}$ over $R \in \mathcal{C} \mathcal{L}$, we denote by $\mathbf{X}^{0}$ the identity component of $\mathbf{X}$. For a map $f: R \rightarrow U \in \mathcal{C} \mathcal{L}$ we denote by $f^{*} \mathbf{X}$ or $\mathbf{X}_{U}$ the base change along $f$.

In $[\mathbf{1 6}]$ we defined the $K(n)$-local commutative ring spectrum $\mathbb{B}$ that is an extension of $E_{n}$. In this section, we introduce a commutative ring spectrum $\mathbb{B}_{n}$ that is equivalent to $\mathbb{B}$ and study a natural $\mathcal{S}_{n+1}$-comodule structure on the $\mathbb{B}_{n}$-cohomology of spaces, which is an extension of the $\mathcal{S}_{n+1}$-comodule structure on the $E_{n+1}$-cohomology of spaces.

\subsection{The $\mathbb{B}_{n}$-theory}

Let $E_{n+1}$ be the $(n+1)$ st Morava $E$-theory. In order to distinguish $E_{n}^{0}$ and $E_{n+1}^{0}$, we write $E_{n+1}^{0}=W\left(\mathbb{F}_{p^{n+1}}\right) \llbracket u_{1}, \ldots, u_{n} \rrbracket$. Let $L_{K(n)}$ be the Bousfield localization functor with respect to $K(n)$. We define the spectrum $\mathbb{A}_{n}$ by

$$
\mathbb{A}_{n}=L_{K(n)} E_{n+1} \text {. }
$$

The spectrum $\mathbb{A}_{n}$ is an even periodic commutative ring spectrum. Note that the degree 0 coefficient ring $\mathbb{A}_{n}^{0}$ is given by

$$
\mathbb{A}_{n}^{0}=\left(W\left(\left(u_{n}\right)\right)\right)_{p}^{\wedge} \llbracket u_{1}, \ldots, u_{n-1} \rrbracket,
$$

where $W=W\left(\mathbb{F}_{p^{n+1}}\right), W\left(\left(u_{n}\right)\right)$ is the ring of formal Laurent series over $W$ with variable $u_{n}$, and $\left(W\left(\left(u_{n}\right)\right)\right)_{p}^{\wedge}$ is the completion of $W\left(\left(u_{n}\right)\right)$ at $p$.

We define the spectrum $\mathbb{B}_{n}$ by

$$
\mathbb{B}_{n}=L_{K(n)}\left(E_{n} \wedge E_{n+1}\right) .
$$

We have ring spectrum maps

$$
\text { inc: } E_{n} \longrightarrow \mathbb{B}_{n} \text {, }
$$

which is given by the inclusion into the first smash factor of $\mathbb{B}_{n}$, and

$$
\operatorname{ch}: E_{n+1} \longrightarrow \mathbb{B}_{n}
$$

which is given by the inclusion into the second smash factor of $\mathbb{B}_{n}$. The map ch induces a map of ring spectra

$$
\mathrm{j}: \mathbb{A}_{n} \longrightarrow \mathbb{B}_{n} .
$$

We use the same symbols for the induced ring homomorphisms on $\pi_{0}$.

Let $\mathbb{F}$ be the composite field of $\mathbb{F}_{p^{n}}$ and $\mathbb{F}_{p^{n+1}}$, that is, the minimal field that contains $\mathbb{F}_{p^{n}}$ and $\mathbb{F}_{p^{n+1}}$. Note that

$$
\mathbb{F} \cong \mathbb{F}_{p^{n}} \otimes_{\mathbb{F}_{p}} \mathbb{F}_{p^{n+1}}
$$

We have the commutative ring spectrum $\mathbb{B}$ constructed in $[\mathbf{1 6}]$. In this paper, we assume that the residue field of the local ring $\pi_{0}(\mathbb{B})$ is $\mathbb{F}$. 
Lemma 4.1. The commutative ring spectrum $\mathbb{B}$ is equivalent to $\mathbb{B}_{n}$.

Proof. For a finite extension $k$ of $\mathbb{F}_{p}$, we denote by $W(k)$ the ring of Witt vectors with coefficients in $k$. Note that we have an isomorphism

$$
W(\mathbb{F}) \cong W\left(\mathbb{F}_{p^{n}}\right) \otimes_{W\left(\mathbb{F}_{p}\right)} W\left(\mathbb{F}_{p^{n+1}}\right) .
$$

Let $S_{p}^{0}$ be the completion of the sphere spectrum $S^{0}$ at $p$. We denote by $S W(k)$ the commutative $S_{p}^{0}$-algebra obtained from $S_{p}^{0}$ by adjoining roots of unity such that the map $S_{p}^{0} \rightarrow S W(k)$ is a $\operatorname{Gal}\left(k / \mathbb{F}_{p}\right)$-Galois extension of commutative $S$-algebras. We set

$$
R(k)=L_{K(n)} S W(k) .
$$

Note that $R\left(\mathbb{F}_{p}\right)=L_{K(n)} S_{p}^{0} \simeq L_{K(n)} S^{0}$. We assume that $L_{K(n)} S^{0}$ is a cofibrant commutative $S$-algebra. We may also assume that the map $L_{K(n)} S^{0} \rightarrow R(k)$ is a $K(n)$ local $\operatorname{Gal}\left(k / \mathbb{F}_{p}\right)$-Galois extension of commutative $S$-algebras. There is an equivalence

$$
R(\mathbb{F}) \simeq R\left(\mathbb{F}_{p^{n}}\right) \wedge_{L_{K(n)} S^{0}} R\left(\mathbb{F}_{p^{n+1}}\right),
$$

of $K(n)$-local commutative $S$-algebras.

Let $E_{n}^{\prime}$ be the variant of the $n$th Morava $E$-theory spectrum such that the residue field of $\left(E_{n}^{\prime}\right)^{0}$ is $\mathbb{F}$ and let $E_{n+1}^{\prime}$ be the variant of the $(n+1)$ st Morava $E$-theory spectrum such that the residue field of $\left(E_{n+1}^{\prime}\right)^{0}$ is $\mathbb{F}$. We have a diagram

$$
E_{n}^{\prime} \longleftarrow R(\mathbb{F}) \longrightarrow L_{K(n)} E_{n+1}^{\prime},
$$

of commutative $S$-algebras by [17, Proposition 3.4]. We assume that $L_{K(n)} E_{n+1}^{\prime}$ and $E_{n}^{\prime}$ are cofibrant commutative $R(\mathbb{F})$-algebras. In $[\mathbf{1 7}]$ we showed that there is an equivalence

$$
\mathbb{B} \simeq L_{K(n)}\left(E_{n}^{\prime} \wedge_{R(\mathbb{F})} L_{K(n)} E_{n+1}^{\prime}\right) .
$$

By [17, Proposition 3.4], we have a canonical map $R\left(\mathbb{F}_{p^{n}}\right) \rightarrow E_{n}$ of commutative $S$ algebras up to homotopy. We may assume that $E_{n}$ is a cofibrant commutative $R\left(\mathbb{F}_{p^{n}}\right)$ algebra. Since $E_{n} \wedge_{R\left(\mathbb{F}_{p^{n}}\right)} R(\mathbb{F})$ is the $K(n)$-local commutative $S$-algebra obtained from $E_{n}$ by adjoining $\left(p^{n+1}-1\right)$ th roots of unity, we see that there is an equivalence

$$
E_{n}^{\prime} \simeq E_{n} \wedge_{R\left(\mathbb{F}_{p^{n}}\right)} R(\mathbb{F}) .
$$

In the same way, by $[\mathbf{1 7}$, Proposition 3.4$]$, we have a canonical map $R\left(\mathbb{F}_{p^{n+1}}\right) \rightarrow$ $L_{K(n)} E_{n+1}$ of commutative $S$-algebras up to homotopy. We may assume that the commutative $S$-algebra $L_{K(n)} E_{n+1}$ is a cofibrant commutative $R\left(\mathbb{F}_{p^{n+1}}\right)$-algebra. Since $L_{K(n)} E_{n+1} \wedge_{R\left(\mathbb{F}_{p^{n+1}}\right)} R(\mathbb{F})$ is the $K(n)$-local commutative $S$-algebra obtained from $L_{K(n)} E_{n+1}$ by adjoining $\left(p^{n}-1\right)$ th roots of unity, we see that there is an equivalence

$$
L_{K(n)} E_{n+1}^{\prime} \simeq L_{K(n)} E_{n+1} \wedge_{R\left(\mathbb{F}_{p^{n+1}}\right)} R(\mathbb{F}) .
$$

By equivalences (1), (2), (3), we obtain an equivalence

$$
E_{n}^{\prime} \wedge_{R(\mathbb{F})} L_{K(n)} E_{n+1}^{\prime} \simeq E_{n} \wedge_{L_{K(n)} S^{0}} L_{K(n)} E_{n+1},
$$

of commutative $S$-algebras. By [5, Lemma 3.13], there is an equivalence

$$
L_{K(n)}\left(E_{n} \wedge_{L_{K(n)} S^{0}} L_{K(n)} E_{n+1}\right) \simeq L_{K(n)}\left(E_{n} \wedge L_{K(n)} E_{n+1}\right) .
$$

Hence we obtain an equivalence between $\mathbb{B}$ and $\mathbb{B}_{n}$. 
Since $E_{n}$ and $E_{n+1}$ are Landweber exact, we have an isomorphism

$$
\pi_{*}\left(E_{n} \wedge E_{n+1}\right) \cong \pi_{*}\left(E_{n}\right) \otimes_{M U_{*}} M U_{*}(M U) \otimes_{M U_{*}} \pi_{*}\left(E_{n+1}\right),
$$

where $M U$ is the complex cobordism spectrum. Since $p, w_{1}, \ldots, w_{n-1}$ is regular in the $\pi_{*}\left(E_{n}\right)$-module $\pi_{*}\left(E_{n} \wedge E_{n+1}\right)$, we see that $\pi_{*}\left(\mathbb{B}_{n}\right)$ is the completion of $\pi_{*}\left(E_{n} \wedge\right.$ $\left.E_{n+1}\right)$ at the ideal $\left(p, w_{1}, \ldots, w_{n-1}\right)$. Taking a coordinate, we can regard $\mathbf{F}_{n}$ as a formal group law over $E_{n}^{0}$ such that its $p$-series is given by

$$
\sum_{i=0}^{n} \mathbf{F}_{n} w_{i} X^{p^{i}},
$$

where $X$ is a formal variable, $w_{0}=p$ and $w_{n}=1$. Also, taking a coordinate, we can regard $\mathbf{F}_{n+1}$ as a formal group law over $E_{n+1}^{0}$ such that its $p$-series is given by

$$
\sum_{i=0}^{n+1} \mathbf{F}_{n+1} u_{i} X^{p^{i}}
$$

where $X$ is a formal variable, $u_{0}=p$ and $u_{n+1}=1$. Note that there is an isomorphism of formal group laws over $\mathbb{B}_{n}^{0}$ between inc ${ }^{*} \mathbf{F}_{n}$ induced by the ring homomorphism inc: $E_{n}^{0} \rightarrow \mathbb{B}_{n}^{0}$ and $\operatorname{ch}^{*} \mathbf{F}_{n+1}$ induced by $\mathrm{ch}: E_{n+1}^{0} \rightarrow \mathbb{B}_{n}^{0}$. The existence of an isomorphism between the two formal group laws implies that $\left(p, w_{1}, \ldots, w_{n-1}\right)=$ $\left(p, u_{1}, \ldots, u_{n-1}\right)$ as ideals of $\pi_{*}\left(\mathbb{B}_{n}\right)$. The spectrum $\mathbb{B}_{n}$ is an even periodic commutative ring spectrum. By $[\mathbf{1 6}, \S 4]$, the degree 0 coefficient ring $\mathbb{B}_{n}^{0}$ is a complete regular local ring with maximal ideal $\left(p, w_{1}, \ldots, w_{n-1}\right)$. Let $L$ be the residue field of $\mathbb{B}_{n}^{0}$. The field $L$ is a Galois extension of $\mathbb{F}_{p}\left(\left(u_{n}\right)\right)$ with Galois group isomorphic to $\operatorname{Gal}\left(\mathbb{F} / \mathbb{F}_{p}\right) \ltimes \mathbb{S}_{n}$, and the fixed field of the subgroup $\mathbb{S}_{n}$ is $\mathbb{F}\left(\left(u_{n}\right)\right)$. Since $\mathbb{F}$ is the composite field of $\mathbb{F}_{p^{n}}$ and $\mathbb{F}_{p^{n+1}}$, we have

$$
\operatorname{Gal}\left(\mathbb{F} / \mathbb{F}_{p}\right) \cong \operatorname{Gal}\left(\mathbb{F}_{p^{n}} / \mathbb{F}_{p}\right) \times \operatorname{Gal}\left(\mathbb{F}_{p^{n+1}} / \mathbb{F}_{p}\right) .
$$

Hence $\operatorname{Gal}\left(\mathbb{F} / \mathbb{F}_{p}\right) \ltimes \mathbb{S}_{n} \cong \operatorname{Gal}\left(\mathbb{F}_{p^{n+1}} / \mathbb{F}_{p}\right) \times \mathbb{G}_{n}$ and $L$ is a Galois extension of the field $\mathbb{F}_{p^{n+1}}\left(\left(u_{n}\right)\right)$ with Galois group isomorphic to $\mathbb{G}_{n}$.

Let $\mathbf{G}=\mathbf{F}_{n+1}\left[p^{\infty}\right]$ be the $p$-divisible group over $E_{n+1}^{0}$ associated to the formal group $\mathbf{F}_{n+1}$. We denote by $\mathbf{G}_{\mathbb{A}}$ the base change along the map $E_{n+1}^{0} \rightarrow \mathbb{A}_{n}^{0}$. The $p$ divisible group associated to the formal group of $\mathbb{A}_{n}$ is identified with the identity component $\mathbf{G}_{\AA}^{0}$. Let $\mathbf{H}=\mathbf{F}_{n}\left[p^{\infty}\right]$ be the $p$-divisible group over $E_{n}^{0}$ associated to the formal group $\mathbf{F}_{n}$. Suppose we have two maps $f: \mathbb{A}_{n}^{0} \rightarrow R$ and $g: E_{n}^{0} \rightarrow R$ in $\mathcal{C} \mathcal{L}$. Then we have two connected $p$-divisible groups $f^{*} \mathbf{G}_{\mathbb{A}}^{0}$ and $g^{*} \mathbf{H}$. We denote by

$$
\operatorname{Iso}\left(f^{*} \mathbf{G}_{\mathbb{A}}^{0}, g^{*} \mathbf{H}\right)
$$

the set of all the isomorphisms of connected $p$-divisible groups between $f^{*} \mathbf{G}_{\mathbb{A}}^{0}$ and $g^{*} \mathbf{H}$, and by

$$
\operatorname{Hom}_{f, g}^{c}\left(\mathbb{B}_{n}^{0}, R\right)
$$

the set of all the maps $h: \mathbb{B}_{n}^{0} \rightarrow R$ in $\mathcal{C} \mathcal{L}$ such that $h \circ \mathrm{j}=f$ and $h \circ$ inc $=g$.

Lemma 4.2. There is a bijection between $\operatorname{Iso}\left(f^{*} \mathbf{G}_{\mathbb{A}}^{0}, g^{*} \mathbf{H}\right)$ and $\operatorname{Hom}_{f, g}^{c}\left(\mathbb{B}_{n}^{0}, R\right)$.

Proof. Let $M U P$ be the periodic complex cobordism spectrum. We have $\pi_{0}\left(E_{n} \wedge\right.$ $\left.\mathbb{A}_{n}\right)=E_{n}^{0} \otimes_{M U P_{0}} M U P_{0}(M U P) \otimes_{M U P_{0}} \mathbb{A}_{n}^{0}$. Since $\mathbb{B}_{n} \simeq L_{K(n)}\left(E_{n} \wedge \mathbb{A}_{n}\right), \mathbb{B}_{n}^{0}$ is the 
completion of $\pi_{0}\left(E_{n} \wedge \mathbb{A}_{n}\right)$ at the ideal $I_{n}=\left(p, w_{1}, \ldots, w_{n-1}\right)$. The lemma follows from the fact that $M U P_{0}(M U P)$ classifies isomorphisms of formal group laws.

\subsection{Finite Subgroups on a $p$-divisible group}

Let $\mathbf{X}$ be a $p$-divisible group over $R \in \mathcal{C} \mathcal{L}$. We assume that the finite subgroup $\mathbf{X}\left[p^{r}\right]$ is embeddable in a smooth curve in the sense of $[\mathbf{9},(1.2 .1)]$ for any $r \geqslant 0$. A divisor on $\mathbf{X}$ is a closed subscheme which is finite and flat over $R$. The degree of a divisor is the rank of the coordinate ring over $R$.

Definition 4.3. A finite subgroup of $\mathbf{X}$ is a divisor which is also a subgroup scheme.

For a finite subgroup $K$, we denote by $\operatorname{deg} K$ the degree of $K$. Note that the degree of a finite subgroup is a power of $p$. We denote by $K^{0}$ the identity component of $K$ and by $\pi_{0}(K)$ the group scheme of connected components. There is an exact sequence

$$
0 \longrightarrow K^{0} \longrightarrow K \longrightarrow \pi_{0}(K) \longrightarrow 0,
$$

of fppf sheaves of abelian groups.

For a nonnegative integer $m$, we define the functor

$$
\operatorname{Sub}(\mathbf{X}, m)
$$

over $\operatorname{Spf}(R)$ by assigning to $U \in \mathcal{C} \mathcal{L}_{R}$ the set of all the finite subgroups of $\mathbf{X}_{U}$ of degree $p^{m}$.

Let $\mathbf{Y}$ be a $p$-divisible group over $T \in \mathcal{C} \mathcal{L}$. If there are a map $f: R \rightarrow T$ in $\mathcal{C} \mathcal{L}$ and an isomorphism

$$
\mathbf{Y} \stackrel{\cong}{\longrightarrow} f^{*} \mathbf{X}
$$

then a finite subgroup of $\mathbf{Y}$ can be regarded as a finite subgroup of $f^{*} \mathbf{X}$. This implies that there is an isomorphism of functors

$$
\operatorname{Sub}(\mathbf{Y}, m) \cong \operatorname{Sub}(\mathbf{X}, m) \times_{\operatorname{Spf}(R)} \operatorname{Spf}(T) .
$$

Now suppose that there is a one dimensional formal Lie group $\mathbf{X}^{\prime}$ of finite height over $R^{\prime} \in \mathcal{C} \mathcal{L}$ and that $\mathbf{X}$ is obtained from the associated $p$-divisible group $\mathbf{X}^{\prime}\left[p^{\infty}\right]$ by base change along a map $R^{\prime} \rightarrow R$. We do not assume that the map $R^{\prime} \rightarrow R$ is a local ring homomorphism. By definition, there is a natural isomorphism

$$
\operatorname{Sub}\left(\mathbf{X}^{\prime}\left[p^{\infty}\right], m\right) \cong \operatorname{Sub}\left(\mathbf{X}^{\prime}, m\right) .
$$

Since $\operatorname{Sub}\left(\mathbf{X}^{\prime}, m\right)$ is represented by $S\left(\mathbf{X}^{\prime}, m\right)$, we obtain the following theorem.

Theorem 4.4. If there is a one dimensional formal Lie group $\mathbf{X}^{\prime}$ of finite height over $R^{\prime} \in \mathcal{C} \mathcal{L}$ and $\mathbf{X}$ is obtained from the associated $p$-divisible group $\mathbf{X}^{\prime}\left[p^{\infty}\right]$ by base change along a map $R^{\prime} \rightarrow R$, then $\operatorname{Sub}(\mathbf{X}, m)$ is represented by the complete Noetherian semilocal ring $S\left(\mathbf{X}^{\prime}, m\right) \otimes_{R^{\prime}} R$ so that

$$
\operatorname{Sub}(\mathbf{X}, m)=\operatorname{Spf}\left(S\left(\mathbf{X}^{\prime}, m\right) \otimes_{R^{\prime}} R\right) .
$$

\subsection{Extensions of $p$-divisible groups}

Suppose there is an exact sequence of $p$-divisible groups

$$
0 \longrightarrow \mathbf{Y} \longrightarrow \mathbf{X} \longrightarrow \mathbf{E} \longrightarrow 0
$$

over $R \in \mathcal{C} \mathcal{L}$ such that $\mathbf{E}$ is étale. For a finite subgroup $K$ of $\mathbf{X}$, we set $L=\mathbf{Y} \times_{\mathbf{X}} K$. 
Notice that $L$ is a finite subgroup of $\mathbf{Y}$ and $K / L$ is a finite subgroup of $\mathbf{E}$. Assigning $K / L$ to $K$, we obtain a map of functors

$$
\pi: \operatorname{Sub}(\mathbf{X}, m) \longrightarrow \coprod_{0 \leqslant r \leqslant m} \operatorname{Sub}(\mathbf{E}, r)
$$

for any $m \geqslant 0$. We identify $A \in \operatorname{Sub}(\mathbf{E}, r)(R)$ with the corresponding map $\operatorname{Spf}(R) \rightarrow$ $\operatorname{Sub}(\mathbf{E}, r)$.

Definition 4.5. For $0 \leqslant r \leqslant m$ and $A \in \operatorname{Sub}(\mathbf{E}, r)(R)$, we define the functor

$$
\operatorname{Sub}(\mathbf{X}, m, A)
$$

over $\operatorname{Spf}(R)$ to be the fiber of $\pi$ at $A$.

Assigning $L$ to $K$, there is a natural transformation

$$
\operatorname{Sub}(\mathbf{X}, m, A) \longrightarrow \operatorname{Sub}(\mathbf{Y}, m-r),
$$

for $0 \leqslant r \leqslant m$ and $A \in \operatorname{Sub}(\mathbf{E}, r)(R)$. Let $0 \in \operatorname{Sub}(\mathbf{E}, 0)(R)$ be the zero divisor. The following lemma is easily obtained.

Lemma 4.6. We have an isomorphism of functors

$$
\operatorname{Sub}(\mathbf{X}, m, 0) \stackrel{\cong}{\rightarrow} \operatorname{Sub}(\mathbf{Y}, m),
$$

for any $m \geqslant 0$.

Lemma 4.7. Let $k$ be an algebraically closed field equipped with a map $R \rightarrow k$ in $\mathcal{C} \mathcal{L}$. If $\mathbf{Y}$ is connected, then $\operatorname{Sub}(\mathbf{X}, m, A)(k)$ is a one-point set for any $m \geqslant 0$ and any $A \in \operatorname{Sub}(\mathbf{E}, r)(R)(0 \leqslant r \leqslant m)$.

Proof. Notice that $\mathbf{Y}$ is isomorphic to the identity component $\mathbf{X}^{0}$ and that $\mathbf{E}$ is isomorphic to the group scheme of connected components $\pi_{0}(\mathbf{X})$. Since $k$ is algebraically closed, exact sequence (4) canonically splits over $k$. Hence we have $\mathbf{X}_{k} \cong \mathbf{Y}_{k} \times \mathbf{E}_{k}$. The lemma follows from the fact that $\operatorname{Sub}(\mathbf{Y}, m-r)(k)$ is a one-point set since $\mathbf{Y}$ is connected.

For an abelian group $M$ and $r \geqslant 0$, we denote by $\operatorname{Sub}(M, r)$ the set of all the subgroups of $M$ with order $p^{r}$. Now suppose $\mathbf{E}$ is constant. Then $\operatorname{Sub}(\mathbf{E}, r)$ is a constant sheaf associated to $\operatorname{Sub}(\mathbf{E}(R), r)$. We identify $A \in \operatorname{Sub}(\mathbf{E}(R), r)$ with the finite subgroup $A_{R}$ of $\mathbf{E}$. There is an isomorphism of functors

$$
\operatorname{Sub}(\mathbf{X}, m) \cong \coprod_{A} \operatorname{Sub}(\mathbf{X}, m, A),
$$

for any $m \geqslant 0$, where $A$ ranges over $\coprod_{r=0}^{m} \operatorname{Sub}(\mathbf{E}(R), r)$.

Theorem 4.8. We suppose that $\mathbf{X}$ is obtained from the $p$-divisible group $\mathbf{X}^{\prime}\left[p^{\infty}\right]$ associated to a one dimensional formal Lie group $\mathbf{X}^{\prime}$ over $R^{\prime} \in \mathcal{C} \mathcal{L}$ by base change along a map $R^{\prime} \rightarrow R$. If $\mathbf{Y}$ is connected and $\mathbf{E}$ is constant, then $\operatorname{Sub}(\mathbf{X}, m, A)$ is represented by a complete Noetherian local ring $S(\mathbf{X}, m, A)$ for any $m \geqslant 0$ and any 
$A \in \coprod_{r=0}^{m} \operatorname{Sub}(\mathbf{E}(R), r)$. There exists a decomposition of $S\left(\mathbf{X}^{\prime}, m\right) \otimes_{R^{\prime}} R$ into a finite product of complete Noetherian local rings

$$
S\left(\mathbf{X}^{\prime}, m\right) \otimes_{R^{\prime}} R=\prod_{A} S(\mathbf{X}, m, A),
$$

for any $m \geqslant 0$, where the product is taken over $A \in \coprod_{r=0}^{m} \operatorname{Sub}(\mathbf{E}(R), r)$.

Proof. By Theorem 4.4, $\operatorname{Sub}(\mathbf{X}, m)$ is represented by $S\left(\mathbf{X}^{\prime}, m\right) \otimes_{R^{\prime}} R$. By $[\mathbf{1 3}$, Theorem 42], $S\left(\mathbf{X}^{\prime}, m\right)$ is finite over $R^{\prime}$. This implies that $S(\mathbf{X}, m) \otimes_{R^{\prime}} R$ is a complete Noetherian semilocal ring. The theorem follows from Lemma 4.7.

For a finite subgroup $K$ of $\mathbf{X}$, we denote by $\mathbf{X} / K$ the quotient as a fppf sheaf of abelian groups. Note that $\mathbf{X} / K$ is a $p$-divisible group by [10, Proposition 2.7]. By the snake lemma, we obtain the following lemma.

Lemma 4.9. There exists an exact sequence

$$
0 \longrightarrow \mathbf{Y} / L \longrightarrow \mathbf{X} / K \longrightarrow \mathbf{E} /(K / L) \longrightarrow 0,
$$

of p-divisible groups over $R$.

\subsection{The decomposition of $S\left(\mathbf{G}_{\mathbb{B}}, m\right)$}

Recall that $\mathbf{G}=\mathbf{F}_{n+1}\left[p^{\infty}\right]$ and $\mathbf{H}=\mathbf{F}_{n}\left[p^{\infty}\right]$. We denote by $\mathbf{G}_{\mathbb{B}}$ the $p$-divisible group over $\mathbb{B}_{n}^{0}$ which is obtained from $\mathbf{G}$ by base change along the map ch: $E_{n+1}^{0} \rightarrow$ $\mathbb{B}_{n}^{0}$, and by $\mathbf{H}_{\mathbb{B}}$ the $p$-divisible group over $\mathbb{B}_{n}^{0}$ which is obtained from $\mathbf{H}$ by base change along the map inc: $E_{n}^{0} \rightarrow \mathbb{B}_{n}^{0}$. The maps ch: $E_{n+1}^{0}\left(\mathbb{C P}^{\infty}\right) \rightarrow \mathbb{B}_{n}^{0}\left(\mathbb{C P}^{\infty}\right)$ and inc: $E_{n}^{0}\left(\mathbb{C P}^{\infty}\right) \rightarrow \mathbb{B}_{n}^{0}\left(\mathbb{C P}^{\infty}\right)$ induce an isomorphism of connected $p$-divisible groups

$$
\theta: \mathbf{G}_{\mathbb{B}}^{0} \cong \mathbf{H}_{\mathbb{B}},
$$

where $\mathbf{G}_{\mathbb{B}}^{0}$ is the identity component of $\mathbf{G}_{\mathbb{B}}$. Furthermore, there is an exact sequence of $p$-divisible groups

$$
0 \rightarrow \mathbf{H}_{\mathbb{B}} \longrightarrow \mathbf{G}_{\mathbb{B}} \longrightarrow\left(\mathbb{Q}_{p} / \mathbb{Z}_{p}\right)_{\mathbb{B}} \rightarrow 0
$$

over $\mathbb{B}_{n}^{0}$ by $[\mathbf{1 8}$, Theorem 5.3].

We set $S\left(\mathbf{G}_{\mathbb{B}}, m, r\right)=S\left(\mathbf{G}_{\mathbb{B}}, m, \mathbb{Q}_{p} / \mathbb{Z}_{p}\left[p^{r}\right]\right)$ for $0 \leqslant r \leqslant m$. For $U \in \mathcal{C} \mathcal{L}_{\mathbb{B}_{n}^{0}}$, we note that $\operatorname{Sub}\left(\mathbf{G}_{\mathbb{B}}, m, r\right)(U)$ is the set of all the finite subgroups $K$ of $\mathbf{G}_{U}$ such that $\operatorname{deg} K=$ $p^{m}$ and $\operatorname{deg} K^{0}=p^{m-r}$. By Theorem 4.8, there is a decomposition

$$
S\left(\mathbf{F}_{n+1}, m\right) \underset{s, E_{n+1}^{0}}{\otimes} \mathbb{B}_{n}^{0} \cong \prod_{r=0}^{m} S\left(\mathbf{G}_{\mathbb{B}}, m, r\right) .
$$

\subsection{Base change of graded Hopf algebroids without antipode}

Let $\Gamma=(E,\{S(m)\}, s, t, i, c)$ be a graded Hopf algebroid without antipode. Suppose $B$ is a commutative ring equipped with a ring homomorphism $\xi: E \rightarrow B$. Set

$$
V(m)=S(m) \otimes_{s, E} B
$$

for each $m \geqslant 0$. We define maps $s_{m}^{\prime}: B \rightarrow V(m)$ for $m \geqslant 0$ and $i^{\prime}: V(0) \rightarrow B$ by $s_{m}^{\prime}=$ $s_{m} \otimes \mathrm{id}_{B}$ and $i^{\prime}=i \otimes \mathrm{id}_{B}$, respectively. We set $\eta_{m}=\operatorname{id}_{S(m)} \otimes \xi: S(m) \cong S(m) \otimes_{s, E}$ 
$E \rightarrow S(m) \otimes_{s, E} B \cong V(m)$ for $m \geqslant 0$. Suppose there is a ring homomorphism

$$
t_{m}^{\prime}: B \rightarrow V(m),
$$

for each $m \geqslant 0$ such that $t_{m}^{\prime} \circ \xi=\eta_{m} \circ t_{m}$. Note that there is an isomorphism

$$
V\left(m^{\prime}\right) \otimes_{s^{\prime}, B, t^{\prime}} V(m) \cong S\left(m^{\prime}\right) \otimes_{s, E, t} S(m) \otimes_{s, E} B,
$$

for any $m, m^{\prime} \geqslant 0$. We define the map $c_{m^{\prime}, m}^{\prime}: V\left(m+m^{\prime}\right) \rightarrow V\left(m^{\prime}\right) \otimes_{s^{\prime}, B, t^{\prime}} V(m)$ for $m, m^{\prime} \geqslant 0$ by $c_{m^{\prime}, m}^{\prime}=c_{m^{\prime}, m} \otimes \operatorname{id}_{B}$. The following lemma is easily obtained.

Lemma 4.10. The data $\Sigma=\left(B,\{V(m)\}, s^{\prime}, t^{\prime}, i^{\prime}, c^{\prime}\right)$ is a graded Hopf algebroid without antipode if and only if $B$ is a commutative $\Gamma$-comodule algebra by the maps $t_{m}^{\prime}: B \rightarrow S(m) \otimes_{s, E} B$ for $m \geqslant 0$. If this holds, then the map $\left(\xi,\left\{\eta_{m}\right\}\right): \Gamma \rightarrow \Sigma$ is a map of graded Hopf algebroids without antipode.

For the remainder of this subsection, we assume that $B$ is a commutative $\Gamma$ comodule algebra by the maps $t_{m}^{\prime}$ for $m \geqslant 0$. Let $R: \operatorname{Mod}(B) \rightarrow \operatorname{Mod}(E)$ be the forgetful functor. We can easily verify that $R$ induces a functor

$$
R: \operatorname{Comod}(\Sigma) \longrightarrow \operatorname{Comod}(\Gamma) .
$$

Let $L: \operatorname{Mod}(E) \rightarrow \operatorname{Mod}(B)$ be the extension of scalars functor given by $L(M)=$ $B \otimes_{E} M$. Since Comod $(\Gamma)$ is a symmetric monoidal category and $B$ is a commutative $\Gamma$-comodule algebra, $R L(M)$ has a $\Gamma$-comodule structure if $M \in \operatorname{Comod}(\Gamma)$.

Lemma 4.11. Let $M$ be a B-module. The map $R(u): R L R(M) \rightarrow R(M)$ is a map of $\Gamma$-comodules if $M$ is a $\Sigma$-comodule, where $u$ is the counit map. Conversely, if $R(M)$ is $a \Gamma$-comodule and $R(u)$ is a map of $\Gamma$-comodules, then $M$ has a $\Sigma$-comodule structure such that $R(M)$ is the given $\Gamma$-comodule.

Proof. Suppose $N$ is a $B$-module and a $\Gamma$-comodule. We can verify that the $\Gamma$ comodule structure maps $N \rightarrow S(m) \otimes_{s, E} N \cong V(m) \otimes_{s^{\prime}, B} N$ for $m \geqslant 0$ give $N$ a $\Sigma$-comodule structure if and only if the multiplication map $B \otimes_{E} N \rightarrow N$ is a map of $\Gamma$-comodules. This completes the proof.

If $M \in \operatorname{Comod}(\Gamma)$, then $R L(M)=B \otimes_{E} M \in \operatorname{Comod}(\Gamma)$. Furthermore, the multiplication map $B \otimes_{E} B \otimes_{E} M \rightarrow B \otimes_{E} M$ is a map of $\Gamma$-comodules since $B$ is a commutative $\Gamma$-comodule algebra. By Lemma 4.11, we see that the functor $L$ extends to a functor

$$
L: \operatorname{Comod}(\Gamma) \longrightarrow \operatorname{Comod}(\Sigma) .
$$

Proposition 4.12. There is an adjoint pair of functors

$$
L: \operatorname{Comod}(\Gamma) \leftrightarrows \operatorname{Comod}(\Sigma): R
$$

where the left adjoint $L$ is the extension of scalars functor and the right adjoint $R$ is the forgetful functor.

Proof. Let $M \in \operatorname{Comod}(\Gamma)$ and $N \in \operatorname{Comod}(\Sigma)$. It is not hard to see that the counit $L R(N) \rightarrow N$ is a map of $\Sigma$-comodules and that the unit $M \rightarrow R L(M)$ is a map of $\Gamma$-comodules. The natural bijection $\operatorname{Hom}_{\operatorname{Mod}(E)}(M, R(N)) \cong \operatorname{Hom}_{\operatorname{Mod}(B)}(L(M), N)$ restricts to a bijection between $\operatorname{Hom}_{\operatorname{Comod}(\Gamma)}(M, R(N))$ and $\operatorname{Hom}_{\operatorname{Comod}(\Sigma)}(L(M), N)$. 
It is not hard to see that $R$ is a symmetric monoidal functor and that $L$ is a strong symmetric monoidal functor. Hence we obtain the following corollary.

Corollary 4.13. There is an adjoint pair of functors

$$
L: \operatorname{ComodAlg}(\Gamma) \leftrightarrows \operatorname{ComodAlg}(\Sigma): R
$$

\subsection{The graded Hopf algebroid $\mathcal{S}_{\mathbb{B}}$ without antipode}

We construct a formal graded Hopf algebroid $\mathcal{S}_{\mathbb{B}}=\left(\mathbb{B}_{n}^{0},\left\{S\left(\mathbf{G}_{\mathbb{B}}, m\right)\right\}, s, t, i, c\right)$ without antipode in this subsection.

Let $s_{m}^{\mathbb{B}}: \mathbb{B}_{n}^{0} \rightarrow S\left(\mathbf{G}_{\mathbb{B}}, m\right)$ be the inclusion for $m \geqslant 0$, and $i^{\mathbb{B}}: S\left(\mathbf{G}_{\mathbb{B}}, 0\right) \rightarrow \mathbb{B}_{n}^{0}$ the canonical isomorphism. There is a decomposition $S\left(\mathbf{G}_{\mathbb{B}}, m\right)=\prod_{r=0}^{m} S\left(\mathbf{G}_{\mathbb{B}}, m, r\right)$ by Theorem 4.8. We denote by $p_{m, r}: S\left(\mathbf{G}_{\mathbb{B}}, m\right) \longrightarrow S\left(\mathbf{G}_{\mathbb{B}}, m, r\right)$ the projection for $0 \leqslant$ $r \leqslant m$. We set $s_{m, r}^{\mathbb{B}}=p_{m, r} \circ s_{m}^{\mathbb{B}}$.

The map ch: $E_{n+1}^{0} \rightarrow \mathbb{B}_{n}^{0}$ induces a map of semilocal rings

$$
\operatorname{ch}(m): S\left(\mathbf{F}_{n+1}, m\right) \longrightarrow S\left(\mathbf{G}_{\mathbb{B}}, m\right),
$$

for $m \geqslant 0$. We define the map

$$
\operatorname{ch}(m, r): S\left(\mathbf{F}_{n+1}, m\right) \longrightarrow S\left(\mathbf{G}_{\mathbb{B}}, m, r\right),
$$

for $0 \leqslant r \leqslant m$ to be the composition $p_{m, r} \circ \operatorname{ch}(m)$. The map $\operatorname{ch}(m, r)$ induces an isogeny of $p$-divisible groups

$$
\operatorname{ch}(m, r)^{*} \Psi_{m}^{E_{n+1}}:\left(s_{m, r}^{\mathbb{B}}\right)^{*} \mathbf{G}_{\mathbb{B}} \longrightarrow \operatorname{ch}(m, r)^{*}\left(t_{m}^{E_{n+1}}\right)^{*} \mathbf{G},
$$

from the isogeny of formal groups $\Psi_{m}^{E_{n+1}}: s_{m}^{*} \mathbf{F}_{n+1} \rightarrow t_{m}^{*} \mathbf{F}_{n+1}$.

Let $S\left(\mathbf{G}_{\mathbb{B}}, m, r\right) \rightarrow R$ be a map in $\mathcal{C} \mathcal{L}$ for $0 \leqslant r \leqslant m$, and let $A_{R}$ be the corresponding finite subgroup of $\mathbf{G}_{R}$. We put $B_{R}=\mathbf{H}_{R} \times \mathbf{G}_{R} A_{R}$. By exact sequence (5), we see that $B_{R}$ is a finite subgroup of $\mathbf{H}_{R}$ with degree $p^{m-r}$, and we obtain a map of local rings

$$
\operatorname{inc}(m, r): S\left(\mathbf{F}_{n}, m-r\right) \longrightarrow S\left(\mathbf{G}_{\mathbb{B}}, m, r\right) .
$$

Notice that $s_{m, r}^{\mathbb{B}} \circ \operatorname{inc}=\operatorname{inc}(m, r) \circ s_{m-r}^{E_{n}}$.

Lemma 4.14. The isomorphism $\theta: \mathbf{G}_{\mathbb{B}}^{0} \cong \mathbf{H}_{\mathbb{B}}$ induces an isomorphism

$$
\bar{\theta}:\left(\mathbf{G}_{R} / A_{R}\right)^{0} \cong \mathbf{H}_{R} / B_{R},
$$

of connected p-divisible groups over $R$.

Proof. This follows from Lemma 4.9 .

Lemma 4.15. The composition $\operatorname{ch}(m, r) \circ t_{m}^{E_{n+1}}: E_{n+1}^{0} \rightarrow S\left(\mathbf{G}_{\mathbb{B}}, m, r\right)$ extends to a map $f: \mathbb{A}_{n}^{0} \rightarrow S\left(\mathbf{G}_{\mathbb{B}}, m, r\right)$ of local rings for any $0 \leqslant r \leqslant m$.

Proof. We have an isomorphism of formal groups $s_{m}^{*} \mathbf{F}_{n+1} / A \cong t_{m}^{*} \mathbf{F}_{n+1}$ over the ring $S\left(\mathbf{F}_{n+1}, m\right)$, where $A$ is the universal finite subgroup of $s_{m}^{*} \mathbf{F}_{n+1}$. This implies that there is an isomorphism of $p$-divisible groups $s_{m}^{*} \mathbf{G} / A \cong t_{m}^{*} \mathbf{G}$. Put $R=S\left(\mathbf{G}_{\mathbb{B}}, m, r\right)$, $h=\operatorname{ch}(m, r) \circ s_{m}$ and $k=\operatorname{ch}(m, r) \circ t_{m}$. We have an isomorphism $h^{*} \mathbf{G} / A_{R} \cong k^{*} \mathbf{G}$. By Lemma 4.14, $\left(h^{*} \mathbf{G} / A_{R}\right)^{0} \cong \mathbf{H}_{R} / B_{R}$, and hence $\left(k^{*} \mathbf{G}\right)^{0} \cong \mathbf{H}_{R} / B_{R}$. Since the height of $\mathbf{H}_{R}$ is $n$, we see that the height of $\left(k^{*} \mathbf{G}\right)^{0}$ is $n$. This implies that $\psi\left(u_{i}\right)=0$ 
$(i<n)$ and $\psi\left(u_{n}\right) \neq 0$ in the residue field of $R$. Hence $k$ extends to a map $f: \mathbb{A}_{n}^{0} \rightarrow R$ since $R$ is a complete local ring.

Proposition 4.16. For any $0 \leqslant r \leqslant m$, there exists a unique map of local rings

$$
t_{m, r}^{\mathbb{B}}: \mathbb{B}_{n}^{0} \longrightarrow S\left(\mathbf{G}_{\mathbb{B}}, m, r\right)
$$

satisfying

$$
\begin{aligned}
& t_{m, r}^{\mathbb{B}} \circ \mathrm{ch}=\operatorname{ch}(m, r) \circ t_{m}^{E_{n+1}}, \\
& t_{m, r}^{\mathbb{B}} \circ \text { inc }=\operatorname{inc}(m, r) \circ t_{m-r}^{E_{n}},
\end{aligned}
$$

and making the following diagram commute

$$
\begin{array}{cc}
\left(s_{m, r}^{\mathbb{B}}\right)^{*} \mathbf{G}_{\mathbb{B}}^{0} \stackrel{\operatorname{ch}(m, r)^{*} \Psi_{m}^{E_{n+1}}}{\longrightarrow}\left(t_{m, r}^{\mathbb{B}}\right)^{*} \mathbf{G}_{\mathbb{B}}^{0} & \downarrow\left(t_{m, r}^{\mathbb{B}}\right)^{*} \theta \\
\left(s_{m, r}^{\mathbb{B}}\right)^{*} \theta \mid & \\
\left(s_{m, r}^{\mathbb{B}}\right)^{*} \mathbf{H}_{\mathbb{B}} \stackrel{\operatorname{inc}(m, r)^{*} \Psi_{m-r}^{E_{n}}}{\longrightarrow}\left(s_{m, r}^{\mathbb{B}}\right)^{*} \mathbf{H}_{\mathbb{B}} .
\end{array}
$$

Proof. Put $R=S\left(\mathbf{G}_{\mathbb{B}}, m, r\right)$. For the extension $f: \mathbb{A}_{n}^{0} \rightarrow R$ of $\operatorname{ch}(m, r) \circ t_{m}^{E_{n+1}}$ given in Lemma 4.15, we have the isogeny $\operatorname{ch}(m, r)^{*} \Psi_{m}^{E_{n+1}}:\left(s_{m, r}^{\mathbb{B}}\right)^{*} \mathbf{G}_{\mathbb{B}}=\mathbf{G}_{R} \rightarrow f^{*} \mathbf{G}_{\mathbb{A}}$. This induces an isomorphism $f^{*} \mathbf{G}_{\mathbb{A}} \cong \mathbf{G}_{R} / A_{R}$, where $A_{R}$ is the universal finite subgroup. For $g=\operatorname{ch}(m, r) \circ t_{m-r}^{E_{n}}$, we have the isogeny $\operatorname{inc}(m, r)^{*} \Psi_{m-r}^{E_{n}}:\left(s_{m, r}^{\mathbb{B}}\right)^{*} \mathbf{H}_{\mathbb{B}}=\mathbf{H}_{R} \rightarrow$ $g^{*} \mathbf{H}_{\mathbb{B}}$. This induces an isomorphism $g^{*} \mathbf{H} \cong \mathbf{H}_{R} / B_{R}$. By Lemma 4.14, the isomorphism $\theta: \mathbf{G}_{\mathbb{B}}^{0} \cong \mathbf{H}_{\mathbb{B}}$ induces an isomorphism $\bar{\theta}: f^{*} \mathbf{G}_{\mathbb{A}}^{0} \cong g^{*} \mathbf{H}$ of connected $p$-divisible groups over $R$. The proposition follows from Lemma 4.2 .

We define the map $t_{m}^{\mathbb{B}}: \mathbb{B}_{n}^{0} \rightarrow S\left(\mathbf{G}_{\mathbb{B}}, m\right)$ for $m \geqslant 0$ by

$$
t_{m}^{\mathbb{B}}=\prod_{r=0}^{m} t_{m, r}^{\mathbb{B}} .
$$

The isogeny of formal groups $\Psi_{m}^{E_{n+1}}: s_{m}^{*} \mathbf{F}_{n+1} \rightarrow t_{m}^{*} \mathbf{F}_{n+1}$ induces an isogeny of $p$ divisible groups

$$
\Psi_{m}^{\mathbb{B}}: s_{m}^{*} \mathbf{G}_{\mathbb{B}} \longrightarrow t_{m}^{*} \mathbf{G}_{\mathbb{B}}
$$

over $S\left(\mathbf{G}_{\mathbb{B}}, m\right)$, which is a disjoint union of the isogenies

$$
\Psi_{m, r}^{\mathbb{B}}: s_{m, r}^{*} \mathbf{G}_{\mathbb{B}} \longrightarrow t_{m, r}^{*} \mathbf{G}_{\mathbb{B}},
$$

for $0 \leqslant r \leqslant m$. Notice that the kernel of $\Psi_{m, r}^{\mathbb{B}}$ is a universal finite subgroup of $s_{m, r}^{*} \mathbf{G}_{\mathbb{B}}$ over $S\left(\mathbf{G}_{\mathbb{B}}, m, r\right)$.

There is an isomorphism between $S\left(\mathbf{F}_{n+1}, m^{\prime}\right) \otimes_{s, E_{n+1}^{0}, t} S\left(\mathbf{F}_{n+1}, m\right) \otimes_{s, E_{n+1}^{0}} \mathbb{B}_{n}^{0}$ and $S\left(\mathbf{G}_{\mathbb{B}}, m^{\prime}\right) \otimes_{s, \mathbb{B}_{n}^{0}, t} S\left(\mathbf{G}_{\mathbb{B}}, m\right)$ for any $m, m^{\prime} \geqslant 0$ by the isomorphism between $S\left(\mathbf{G}_{\mathbb{B}}, m\right)$ and $S\left(\mathbf{F}_{n+1}, m\right) \otimes_{s, E_{n+1}^{0}} \mathbb{B}_{n}^{0}$ for $m \geqslant 0$. We define the map

$$
c_{m^{\prime}, m}^{\mathbb{B}}: S\left(\mathbf{G}_{\mathbb{B}}, m+m^{\prime}\right) \longrightarrow S\left(\mathbf{G}_{\mathbb{B}}, m^{\prime}\right) \underset{s, \mathbb{B}_{n}^{0}, t}{\otimes} S\left(\mathbf{G}_{\mathbb{B}}, m\right),
$$

for $m, m^{\prime} \geqslant 0$ by $c_{m^{\prime}, m}^{\mathbb{B}}=c_{m^{\prime}, m}^{E_{n+1}} \otimes \mathrm{id}_{\mathbb{B}_{n}^{0}}$. 
Lemma 4.17. The map $c_{m^{\prime}, m}^{\mathbb{B}}$ induces a map of local rings

$$
c_{m^{\prime}, m, r^{\prime}, r}^{\mathbb{B}}: S\left(\mathbf{G}_{\mathbb{B}}, m+m^{\prime}, r+r^{\prime}\right) \longrightarrow S\left(\mathbf{G}_{\mathbb{B}}, m^{\prime}, r^{\prime}\right) \underset{s, \mathbb{B}_{n}^{0}, t}{\otimes} S\left(\mathbf{G}_{\mathbb{B}}, m, r\right),
$$

for any $0 \leqslant r \leqslant m$ and any $0 \leqslant r^{\prime} \leqslant m^{\prime}$.

Proof. Suppose there are isogenies of $p$-divisible groups $f: G_{1} \rightarrow G_{2}$ and $g: G_{2} \rightarrow$ $G_{3}$ over $R \in \mathcal{C} \mathcal{L}$ such that $\operatorname{deg} \operatorname{ker}(f)=p^{m}$ and $\operatorname{deg} \operatorname{ker}(g)=p^{m^{\prime}}$. If $\operatorname{deg} \operatorname{ker}(f)^{0}=$ $p^{m-r}$ and $\operatorname{deg} \operatorname{ker}(g)^{0}=p^{m^{\prime}-r^{\prime}}$, then $\operatorname{deg} \operatorname{ker}(g \circ f)^{0}=p^{m+m^{\prime}-r-r^{\prime}}$. Hence the map $\left(p_{m^{\prime}, r^{\prime}} \otimes p_{m, r}\right) \circ c_{m^{\prime}, m}^{\mathbb{B}}$ factors through $S\left(\mathbf{G}_{\mathbb{B}}, m+m^{\prime}, r+r^{\prime}\right)$.

The following lemma is easily obtained.

Lemma 4.18. There is a commutative diagram

$$
\begin{aligned}
& S\left(\mathbf{F}_{n}, m+m^{\prime}-r-r^{\prime}\right) \stackrel{c_{m^{\prime}-r^{\prime}, m-r}^{E_{n}}}{\longrightarrow} S\left(\mathbf{F}_{n}, m^{\prime}-r^{\prime}\right) \underset{s, E_{n}^{0}, t}{\otimes} S\left(\mathbf{F}_{n}, m-r\right) \\
&\left.\operatorname{inc}\left(m+m^{\prime}, r+r^{\prime}\right)\right\rfloor \mid \operatorname{inc}\left(m^{\prime}, r^{\prime}\right) \otimes \operatorname{inc}(m, r) \\
& S\left(\mathbf{G}_{\mathbb{B}}, m+m^{\prime}, r+r^{\prime}\right) \stackrel{c_{m^{\prime}, m, r^{\prime}, r}}{\longrightarrow} S\left(\mathbf{G}_{\mathbb{B}}, m^{\prime}, r^{\prime}\right) \underset{s, \mathbb{B}_{n}^{0}, t}{\otimes} S\left(\mathbf{G}_{\mathbb{B}}, m, r\right),
\end{aligned}
$$

for any $0 \leqslant r \leqslant m$ and any $0 \leqslant r^{\prime} \leqslant m^{\prime}$.

Proposition 4.19. There is a commutative diagram

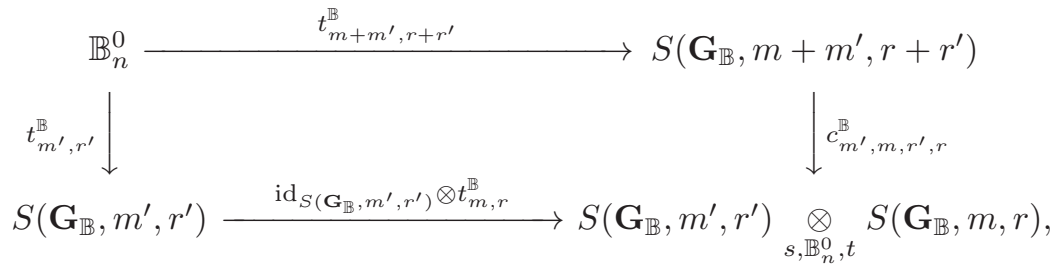

for any $0 \leqslant r \leqslant m$ and any $0 \leqslant r^{\prime} \leqslant m^{\prime}$.

Proof. This is obtained by Lemma 4.2, Proposition 4.16, and Lemma 4.18.

Corollary 4.20. The maps $t_{m}^{\mathbb{B}}: \mathbb{B}_{n}^{0} \rightarrow S\left(\mathbf{F}_{n+1}, m\right) \otimes_{s, E_{n+1}^{0}} \mathbb{B}_{n}^{0}$ make $\mathbb{B}_{n}^{0}$ into a commutative $\mathcal{S}_{n+1}$-comodule algebra.

We use abbreviations $s, t, i, c$ for $s_{m}^{\mathbb{B}}, t_{m}^{\mathbb{B}}, i^{\mathbb{B}}, c_{m^{\prime}, m}^{\mathbb{B}}\left(m, m^{\prime} \geqslant 0\right)$, respectively, when there is no confusion. By Lemma 4.10, we obtain the following theorem.

Theorem 4.21. The data $\mathcal{S}_{\mathbb{B}}=\left(\mathbb{B}_{n}^{0},\left\{S\left(\mathbf{G}_{\mathbb{B}}, m\right)\right\}, s, t, i, c\right)$ represents a formal graded affine category scheme. The map ch induces a map ch: $\mathcal{S}_{n+1} \rightarrow \mathcal{S}_{\mathbb{B}}$ of graded Hopf algebroids without antipode.

By Proposition 4.12 and Corollary 4.13, we obtain the following proposition. 
Proposition 4.22. There is an adjoint pair of functors

$$
L_{n+1}: \operatorname{Comod}\left(\mathcal{S}_{n+1}\right) \leftrightarrows \operatorname{Comod}\left(\mathcal{S}_{\mathbb{B}}\right): R_{n+1}
$$

where the left adjoint $L_{n+1}$ is the extension of scalars functor given by $L_{n+1}(M)=$ $\mathbb{B}_{n}^{0} \otimes_{E_{n+1}^{0}} M$ for $M \in \operatorname{Comod}\left(\mathcal{S}_{n+1}\right)$ and the right adjoint $R_{n+1}$ is the forgetful functor. This induces an adjoint pair of functors

$$
L_{n+1}: \operatorname{Comod} \operatorname{Alg}\left(\mathcal{S}_{n+1}\right) \leftrightarrows \operatorname{Comod} \operatorname{Alg}\left(\mathcal{S}_{\mathbb{B}}\right): R_{n+1}
$$

\section{Comparison of comodules over the Hopf algebroids}

In this section, we construct a functor which compares the category of $\mathcal{S}_{n+1^{-}}$ comodules with compatible $\mathbb{G}_{n+1}$-action and the category of $\mathcal{S}_{n}$-comodules with compatible $\mathbb{G}_{n}$-action.

\subsection{The group $\mathbb{G}_{n, n+1}$}

Recall that $\mathbb{F}$ is the composite field of $\mathbb{F}_{p^{n}}$ and $\mathbb{F}_{p^{n+1}}$ in this paper. In $[\mathbf{1 7}, \S 5]$ we constructed a commutative $S$-algebra $\mathbb{C}$ and the action of the profinite group

$$
\mathbb{G}=\left(\operatorname{Gal}\left(\mathbb{F} / \mathbb{F}_{p}\right) \ltimes \mathbb{S}_{n}\right) \times_{\operatorname{Gal}\left(\mathbb{F} / \mathbb{F}_{p}\right)}\left(\operatorname{Gal}\left(\mathbb{F} / \mathbb{F}_{p}\right) \ltimes \mathbb{S}_{n+1}\right)
$$

on $\mathbb{C}$ in the category of commutative $S$-algebras (see [17, Lemma 5.3]). We set

$$
\mathbb{G}_{n, n+1}=\mathbb{G}_{n} \times \mathbb{G}_{n+1} .
$$

There is an isomorphism

$$
\left(\operatorname{Gal}\left(\mathbb{F} / \mathbb{F}_{p}\right) \ltimes \mathbb{S}_{n}\right) \times \operatorname{Gal}\left(\mathbb{F} / \mathbb{F}_{p}\right)\left(\operatorname{Gal}\left(\mathbb{F} / \mathbb{F}_{p}\right) \ltimes \mathbb{S}_{n+1}\right) \cong \operatorname{Gal}\left(\mathbb{F} / \mathbb{F}_{p}\right) \ltimes\left(\mathbb{S}_{n} \times \mathbb{S}_{n+1}\right) .
$$

By the isomorphism $\operatorname{Gal}\left(\mathbb{F} / \mathbb{F}_{p}\right) \cong \operatorname{Gal}\left(\mathbb{F}_{p^{n}} / \mathbb{F}_{p}\right) \times \operatorname{Gal}\left(\mathbb{F}_{p^{n+1}} / \mathbb{F}_{p}\right)$, we obtain an isomorphism

$$
\mathbb{G} \cong \mathbb{G}_{n, n+1} .
$$

Hence $\mathbb{G}_{n, n+1}$ acts on $\mathbb{C}$ in the category of commutative $S$-algebras. The commutative $S$-algebra $\mathbb{C}$ is a model of the ring spectrum $\mathbb{B}$ constructed in $[\mathbf{1 6}]$ by $[\mathbf{1 7}$, Theorems 4.1 and 5.4]. Note that the spectrum $\mathbb{B}$ in $[\mathbf{1 6}]$ is the spectrum $\overline{\mathbb{B}}$ in $[\mathbf{1 7}]$. Hence we may think that $\mathbb{B}$ is a commutative $S$-algebra and the group $\mathbb{G}_{n, n+1}$ acts on $\mathbb{B}$ in the category of commutative $S$-algebras.

By the work of Goerss-Hopkins in [6], we have the action of $\mathbb{G}_{n}$ on $E_{n}$ and the action of $\mathbb{G}_{n+1}$ on $E_{n+1}$ in the category of commutative $S$-algebras. These two actions induce an action of $\mathbb{G}_{n, n+1}$ on $\mathbb{B}_{n}=L_{K(n)}\left(E_{n} \wedge E_{n+1}\right)$ in the category of commutative $S$-algebras. We see that the actions of $\mathbb{G}_{n, n+1}$ on $\mathbb{B}$ and on $\mathbb{B}_{n}$ are compatible on the point-set level under the equivalence in Lemma 4.1 by the following lemma.

Lemma 5.1. There is an equivalence $\mathbb{B}_{n} \stackrel{\simeq}{\rightrightarrows} \mathbb{B}$ of commutative $S$-algebras, which is $\mathbb{G}_{n, n+1}$-equivariant on the point-set level.

Proof. We use the notation in the proof of Lemma 4.1. We regard the commutative $S$-algebra $\mathbb{C}$ as a model of the ring spectrum $\mathbb{B}$. By definition, we have

$$
\mathbb{C}=L_{K(n)}\left(E_{n}^{\prime} \wedge_{R(\mathbb{F})} L_{K(n)} E_{n+1}^{\prime}\right)
$$

(see $[\mathbf{1 7}, \S 5])$. The group $\mathbb{G}$ acts on $E_{n}^{\prime}$ through the projection $\mathbb{G} \rightarrow \operatorname{Gal}\left(\mathbb{F} / \mathbb{F}_{p}\right) \ltimes \mathbb{S}_{n}$, 
on $L_{K(n)} E_{n+1}^{\prime}$ through $\mathbb{G} \rightarrow \operatorname{Gal}\left(\mathbb{F} / \mathbb{F}_{p}\right) \ltimes \mathbb{S}_{n+1}$, and on $R(\mathbb{F})$ through $\mathbb{G} \rightarrow \operatorname{Gal}\left(\mathbb{F} / \mathbb{F}_{p}\right)$ in the category of commutative $S$-algebras. Since the maps $R(\mathbb{F}) \rightarrow E_{n}^{\prime}$ and $R(\mathbb{F}) \rightarrow$ $L_{K(n)} E_{n+1}^{\prime}$ are $\mathbb{G}$-equivariant, the group $\mathbb{G} \cong \mathbb{G}_{n, n+1}$ acts on $\mathbb{C}$ in the category of commutative $S$-algebras.

By (2), we have the equivalence $E_{n}^{\prime} \simeq E_{n} \wedge_{R\left(\mathbb{F}_{p^{n}}\right)} R(\mathbb{F})$ of commutative $S$-algebras. By the inclusion into the left factor, we obtain a map $E_{n} \rightarrow E_{n}^{\prime}$ of commutative $S$ algebras. This map is $\mathbb{G}$-equivariant on the point-set level, where $\mathbb{G}$ acts on $E_{n}$ through the projection $\mathbb{G} \cong \mathbb{G}_{n, n+1} \rightarrow \mathbb{G}_{n}$. By $(3)$, we have the equivalence $L_{K(n)} E_{n+1}^{\prime} \simeq$ $L_{K(n)} E_{n+1} \wedge_{R\left(\mathbb{F}_{p^{n+1}}\right)} R(\mathbb{F})$ of commutative $S$-algebras. By the inclusion into the left factor after the $K(n)$-localization, we obtain a map $E_{n+1} \rightarrow L_{K(n)} E_{n+1}^{\prime}$ of commutative $S$-algebras. This map is $\mathbb{G}$-equivariant on the point-set level, where $\mathbb{G}$ acts on $E_{n+1}$ through the projection $\mathbb{G} \cong \mathbb{G}_{n, n+1} \rightarrow \mathbb{G}_{n+1}$. These two maps induce a map

$$
\mathbb{B}_{n}=L_{K(n)}\left(E_{n} \wedge E_{n+1}\right) \longrightarrow L_{K(n)}\left(E_{n}^{\prime} \wedge_{R(\mathbb{F})} L_{K(n)} E_{n+1}^{\prime}\right)=\mathbb{C}
$$

of commutative $S$-algebras. By Lemma 4.1 and its proof, this map is an equivalence of commutative $S$-algebras. By the construction, we see that this map is equivariant with respect to the actions of $\mathbb{G} \cong \mathbb{G}_{n, n+1}$ on both sides.

By [17, Theorem 5.6], the action of $\mathbb{G}$ on $\mathbb{C}$ in the category of commutative $S$ algebras is a lifting of the action on $\mathbb{B}$ in the stable homotopy category. In particular, the induced action of $\mathbb{G}_{n, n+1}$ on the commutative ring $\mathbb{B}_{n}^{0}$ coincides with the action on $\mathbb{B}^{0}$ considered in $[\mathbf{1 6}]$ (see [16, Theorem 4.15] for the action of $\mathbb{G}$ on the cohomology theory $\left.\mathbb{B}^{*}(-)\right)$. Now we shall consider the category

$$
\mathbb{G}_{n, n+1}-\operatorname{Mod}\left(\mathbb{B}_{n}^{0}\right)
$$

of twisted $\mathbb{B}_{n}^{0}-\mathbb{G}_{n, n+1}$-modules.

By $\left[\mathbf{1 6}\right.$, Corollary 6.4], the fixed subring of $\mathbb{B}_{n}^{0}$ under $\mathbb{S}_{n+1}$ is isomorphic to $\left(E_{n}^{\prime}\right)^{0}$ :

$$
\left(\mathbb{B}_{n}^{0}\right)^{\mathbb{S}_{n+1}} \cong\left(E_{n}^{\prime}\right)^{0} .
$$

We note that $\mathbb{S}_{n+1}$ is a normal subgroup of $\mathbb{G}_{n, n+1}$ and there is an isomorphism $\mathbb{G}_{n, n+1} / \mathbb{S}_{n+1} \cong \mathbb{G}_{n} \times \operatorname{Gal}\left(\mathbb{F}_{p^{n+1}} / \mathbb{F}_{p}\right)$. Hence the group $\mathbb{G}_{n} \times \operatorname{Gal}\left(\mathbb{F}_{p^{n+1}} / \mathbb{F}_{p}\right)$ acts on the commutative ring $\left(E_{n}^{\prime}\right)^{0}$.

Since

$$
\begin{aligned}
\left(E_{n}^{\prime}\right)^{0} & \cong E_{n}^{0} \otimes_{W\left(\mathbb{F}_{p^{n}}\right)} W(\mathbb{F}) \\
& \cong E_{n}^{0} \otimes_{W\left(\mathbb{F}_{p}\right)} W\left(\mathbb{F}_{p^{n+1}}\right)
\end{aligned}
$$

and the inclusion map $W\left(\mathbb{F}_{p}\right) \rightarrow W\left(\mathbb{F}_{p^{n+1}}\right)$ is a Galois extension of commutative rings with Galois group $\operatorname{Gal}\left(\mathbb{F}_{p^{n+1}} / \mathbb{F}_{p}\right)$, the inclusion map $E_{n}^{0} \rightarrow\left(E_{n}^{\prime}\right)^{0}$ is also a Galois extension of commutative rings with Galois group $\operatorname{Gal}\left(\mathbb{F}_{p^{n+1}} / \mathbb{F}_{p}\right)$ (see, for example, $[\mathbf{7}$, Lemma 1.11]). In particular, we obtain an isomorphism

$$
\left(\left(E_{n}^{\prime}\right)^{0}\right)^{\operatorname{Gal}\left(\mathbb{F}_{p^{n+1}} / \mathbb{F}_{p}\right)} \cong E_{n}^{0} .
$$

Hence we see that the map inc induces an isomorphism

$$
E_{n}^{0} \stackrel{\cong}{\longrightarrow}\left(\mathbb{B}_{n}^{0}\right)^{\mathbb{G}_{n+1}} .
$$

Thus, we can define the functor

$$
F_{n}: \mathbb{G}_{n, n+1}-\operatorname{Mod}\left(\mathbb{B}_{n}^{0}\right) \longrightarrow \mathbb{G}_{n}-\operatorname{Mod}\left(E_{n}^{0}\right)
$$


by assigning the $\mathbb{G}_{n+1}$-invariant submodule $M^{\mathbb{G}_{n+1}}$ to a twisted $\mathbb{B}_{n}^{0}$ - $\mathbb{G}_{n, n+1}$-module $M$. We can easily see that the extension of scalars functor $L_{n+1}: \operatorname{Mod}\left(E_{n+1}^{0}\right) \rightarrow$ $\operatorname{Mod}\left(\mathbb{B}_{n}^{0}\right)$ extends to a functor

$$
L_{n+1}: \mathbb{G}_{n+1}-\operatorname{Mod}\left(E_{n+1}^{0}\right) \longrightarrow \mathbb{G}_{n, n+1}-\operatorname{Mod}\left(\mathbb{B}_{n}^{0}\right) .
$$

We define the functor

$$
C: \mathbb{G}_{n+1}-\operatorname{Mod}\left(E_{n+1}^{0}\right) \longrightarrow \mathbb{G}_{n}-\operatorname{Mod}\left(E_{n}^{0}\right)
$$

to be the composition $F_{n} L_{n+1}$. The functor $C$ induces a functor

$$
C: \mathbb{G}_{n+1}-\operatorname{CAlg}\left(E_{n+1}^{0}\right) \longrightarrow \mathbb{G}_{n}-\operatorname{CAlg}\left(E_{n}^{0}\right) .
$$

For any spectrum $X$, we have $E_{n}^{0}(X) \in \mathbb{G}_{n}-\operatorname{Mod}\left(E_{n}^{0}\right)$. Furthermore, if $X$ is a space, then $E_{n}^{0}(X) \in \mathbb{G}_{n}-\mathrm{CAlg}\left(E_{n}^{0}\right)$.

Lemma 5.2. The map inc: $E_{n}^{0}(X) \rightarrow \mathbb{B}_{n}^{0}(X)$ induces a natural isomorphism

$$
E_{n}^{0}(X) \stackrel{\cong}{\longrightarrow} F_{n}\left(\mathbb{B}_{n}^{0}(X)\right)
$$

in $\mathbb{G}_{n}-\operatorname{Mod}\left(E_{n}^{0}\right)$ for any spectrum $X$. If $X$ is a space, then this is an isomorphism in $\mathbb{G}_{n}-\mathrm{CAlg}\left(E_{n}^{0}\right)$.

Proof. By [16, Theorem A], the inclusion map $\left(E_{n}^{\prime}\right)^{0}(X) \rightarrow \mathbb{B}_{n}^{0}(X)$ induces a natural isomorphism

$$
\left(E_{n}^{\prime}\right)^{0}(X) \stackrel{\cong}{\longrightarrow} \mathbb{B}_{n}^{0}(X)^{\mathbb{S}_{n+1}},
$$

in $\left(\operatorname{Gal}\left(\mathbb{F} / \mathbb{F}_{p}\right) \ltimes \mathbb{S}_{n}\right)-\operatorname{Mod}\left(\left(E_{n}^{\prime}\right)^{0}\right)$ for any spectrum $X$. Note that $\operatorname{Gal}\left(\mathbb{F} / \mathbb{F}_{p}\right) \ltimes \mathbb{S}_{n} \cong$

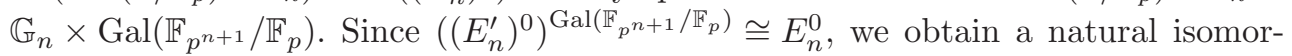
phism

$$
\left(E_{n}^{\prime}\right)^{0}(X)^{\operatorname{Gal}\left(\mathbb{F}_{p^{n+1}} / \mathbb{F}_{p}\right)} \cong \mathbb{B}_{n}^{0}(X)^{\mathbb{G}_{n+1}},
$$

of twisted $E_{n}^{0}-\mathbb{G}_{n}$-modules. Since

$$
\begin{aligned}
\left(E_{n}^{\prime}\right)^{0}(X) & \cong E_{n}^{0}(X) \otimes_{W\left(\mathbb{F}_{p^{n}}\right)} W(\mathbb{F}) \\
& \cong E_{n}^{0}(X) \otimes_{W\left(\mathbb{F}_{p}\right)} W\left(\mathbb{F}_{p^{n+1}}\right)
\end{aligned}
$$

and the inclusion map $W\left(\mathbb{F}_{p}\right) \rightarrow W\left(\mathbb{F}_{p^{n+1}}\right)$ is a Galois extension of commutative rings with Galois group $\operatorname{Gal}\left(\mathbb{F}_{p^{n+1}} / \mathbb{F}_{p}\right)$, we see that the inclusion map $E_{n}^{0}(X) \rightarrow\left(E_{n}^{\prime}\right)^{0}(X)$ induces a natural isomorphism

$$
\left(E_{n}^{\prime}\right)^{0}(X)^{\operatorname{Gal}\left(\mathbb{F}_{p^{n+1}} / \mathbb{F}_{p}\right)} \cong E_{n}^{0}(X)
$$

(see, for example, $\left[\mathbf{7}\right.$, Theorem 7.1]). Hence we see that the map inc: $E_{n}^{0}(X) \rightarrow \mathbb{B}_{n}^{0}(X)$ induces a natural isomorphism

$$
E_{n}^{0}(X) \stackrel{\cong}{\longrightarrow} F_{n}\left(\mathbb{B}_{n}^{0}(X)\right)
$$

in $\mathbb{G}_{n}-\operatorname{Mod}\left(E_{n}^{0}\right)$ for any spectrum $X$. If $X$ is a space, then this is an isomorphism in $\mathbb{G}_{n}$-CAlg $\left(E_{n}^{0}\right)$ since inc: $E_{n}^{0}(X) \rightarrow \mathbb{B}_{n}^{0}(X)$ is a map of commutative rings.

Let $M U P$ be the periodic complex cobordism spectrum. When $X$ is a finite spectrum, we have isomorphisms $E_{n+1}^{0}(X) \cong E_{n+1}^{0} \otimes_{M U P^{0}} M U P^{0}(X)$ and $\mathbb{B}_{n}^{0}(X) \cong$ 
$\mathbb{B}_{n}^{0} \otimes_{M U P^{0}} M U P^{0}(X) \cong \mathbb{B}_{n}^{0} \otimes_{E_{n+1}^{0}} E_{n+1}^{0} \otimes_{M U P^{0}} M U P^{0}(X)$. Hence there is a natural isomorphism $\mathbb{B}_{n}^{0}(X) \cong \mathbb{B}_{n}^{0} \otimes_{E_{n+1}^{0}} E_{n+1}^{0}(X)$. This implies that there is a natural isomorphism

$$
E_{n}^{0}(X) \cong C\left(E_{n+1}^{0}(X)\right)
$$

in $\mathbb{G}_{n}-\operatorname{Mod}\left(E_{n}^{0}\right)$ for any finite spectrum $X$. If $X$ is a finite complex, then this is an isomorphism in $\mathbb{G}_{n}-\mathrm{CAlg}\left(E_{n}^{0}\right)$.

\subsection{Group action on the base change of a Hopf algebroid}

We let $\Gamma=(E,\{S(m)\}, s, t, i, c)$ be a graded Hopf algebroid without antipode equipped with an action of a group $G$. Suppose that there is a map $\xi: E \rightarrow B$ of commutative rings and that, as in Lemma 4.10, we can form a graded Hopf algebroid $\Sigma=\left(B,\{V(m)\}, s^{\prime}, t^{\prime}, i^{\prime}, c^{\prime}\right)$ without antipode. If $G$ acts on $B$ and $\xi$ is $G$-equivariant, then $G$ acts diagonally on $V(m)=S(m) \otimes_{s, E} B$ for $m \geqslant 0$. The following lemma is easily obtained.

Lemma 5.3. If $t_{m}^{\prime}: B \rightarrow V(m)$ is $G$-equivariant for all $m \geqslant 0$, then $G$ acts on the graded Hopf algebroid $\Sigma$ without antipode and the map $\left(\xi,\left\{\operatorname{id}_{S(m)} \otimes \xi\right\}\right): \Gamma \rightarrow \Sigma$ of graded Hopf algebroids without antipode is G-equivariant.

For the remainder of this subsection, we assume that $G$ acts on $B$ and that $\xi: E \rightarrow$ $B$ and $t_{m}^{\prime}: B \rightarrow V(m)$ for all $m \geqslant 0$ are $G$-equivariant.

Let $H$ be a normal subgroup of $G$. If the restriction of the $G$-action to $H$ is trivial on $E$, then there is an adjoint pair of functors

$$
L:(G / H)-\operatorname{Mod}(E) \leftrightarrows G-\operatorname{Mod}(B): F
$$

where the left adjoint $L$ is the extension of scalars functor and the right adjoint $F$ assigns to $N \in G$-Mod $(B)$ the $H$-invariant submodule $N^{H}$.

We suppose that the restriction of the $G$-action to $H$ is trivial on $S(m)$ for all $m \geqslant 0$. This implies that $H$ acts trivially on $E$. Furthermore, we suppose that $S(m)$ is a projective module over $E$ via $s_{m}$ for all $m \geqslant 0$. If $F$ is a free $E$-module with trivial $H$ action and $N$ is a twisted $E$ - $H$-module, then $F \otimes_{E} N$ is isomorphic to the coproduct of copies of $N$ as twisted $E$ - $H$-modules. Hence we have $\left(F \otimes_{E} N\right)^{H} \cong F \otimes_{E} N^{H}$. If $P$ is a projective $E$-module with trivial $H$-action, then $\left(P \otimes_{E} N\right)^{H} \cong P \otimes_{E} N^{H}$ since $P$ is a direct summand for some free $E$-module with trivial $H$-action. Hence we see that there is a natural isomorphism

$$
\left(S(m) \otimes_{s, E} N\right)^{H} \cong S(m) \otimes_{s, E} N^{H},
$$

for any twisted $E$ - $H$-module $N$ and any $m \geqslant 0$. We can extend Proposition 4.12 and Corollary 4.13 as follows.

Proposition 5.4. Let $\Gamma=(E,\{S(m)\}, s, t, i, c)$ be a graded Hopf algebroid without antipode equipped with an action of a group $G$. We assume that $S(m)$ is a projective module over $E$ via $s_{m}$ for all $m \geqslant 0$. We also assume that there is a map $\xi: E \rightarrow B$ of commutative rings and that we can form a graded Hopf algebroid $\Sigma=$ $\left(B,\{V(m)\}, s^{\prime}, t^{\prime}, i^{\prime}, c^{\prime}\right)$ without antipode as in Lemma 4.10. Furthermore, we assume that the map $\xi$ and $t_{m}^{\prime}: B \rightarrow V(m)$ for all $m \geqslant 0$ are $G$-equivariant. 
If a normal subgroup $H$ of $G$ acts trivially on $S(m)$ for all $m \geqslant 0$, then there is an adjoint pair of functors

$$
L:(G / H)-\operatorname{Comod}(\Gamma) \leftrightarrows G-\operatorname{Comod}(\Sigma): F
$$

where the left adjoint $L$ is the extension of scalars functor and the right adjoint $F$ is given by $F(N)=N^{H}$ for $N \in G$-Comod $(\Sigma)$. This induces an adjoint pair of functors

$$
L:(G / H) \text {-ComodAlg }(\Gamma) \leftrightarrows G \text {-ComodAlg }(\Sigma): F
$$

\subsection{The $\mathbb{G}_{n, n+1}$-action on $\mathcal{S}_{\mathbb{B}}$}

The group $\mathbb{G}_{n, n+1}$ acts on exact sequence (5) and hence we have a map of exact sequences

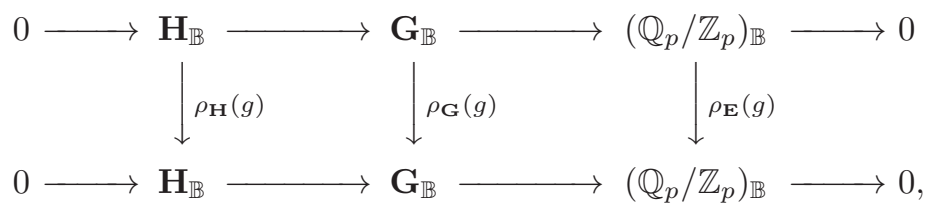

for $g \in \mathbb{G}_{n, n+1}$ covering the action on $\mathbb{B}_{n}^{0}$. Note that $\rho_{\mathbf{H}}(g)$ is the identity map for $g \in \mathbb{G}_{n+1}$ and that $\rho_{\mathbf{G}}(g)$ is the identity map for $g \in \mathbb{G}_{n}$ (see $[\mathbf{1 6}, \S 4]$ ). This induces an action of $\mathbb{G}_{n, n+1}$ on $S\left(\mathbf{G}_{\mathbb{B}}, m\right)$ for $m \geqslant 0$. In particular, we obtain an action of $\mathbb{G}_{n, n+1}$ on $S\left(\mathbf{G}_{\mathbb{B}}, m, r\right)$ for $0 \leqslant r \leqslant m$ such that the projection $p_{m, r}: S\left(\mathbf{G}_{\mathbb{B}}, m\right) \rightarrow S\left(\mathbf{G}_{\mathbb{B}}, m, r\right)$ is $\mathbb{G}_{n, n+1}$-equivariant.

Recall that there is an isomorphism $S\left(\mathbf{G}_{\mathbb{B}}, m\right) \cong S\left(\mathbf{F}_{n+1}, m\right) \otimes_{s, E_{n+1}^{0}} \mathbb{B}_{n}^{0}$ for any $m \geqslant 0$. We suppose that $\mathbb{G}_{n, n+1}$ acts diagonally on $S\left(\mathbf{F}_{n+1}, m\right) \otimes_{s, E_{n+1}^{0}} \mathbb{B}_{n}^{0}$, where the action on $S\left(\mathbf{F}_{n+1}, m\right)$ is given through the projection $\mathbb{G}_{n, n+1} \rightarrow \mathbb{G}_{n+1}$. The following lemma is easily obtained.

Lemma 5.5. For any $m \geqslant 0$, the isomorphism $S\left(\mathbf{G}_{\mathbb{B}}, m\right) \cong S\left(\mathbf{F}_{n+1}, m\right) \otimes_{s, E_{n+1}^{0}} \mathbb{B}_{n}^{0}$ respects the $\mathbb{G}_{n, n+1}$-actions.

By Lemma 4.6, we have an isomorphism $S\left(\mathbf{G}_{\mathbb{B}}, m, 0\right) \cong S\left(\mathbf{F}_{n}, m\right) \otimes_{s, E_{n}^{0}} \mathbb{B}_{n}^{0}$ for any $m \geqslant 0$. We suppose that $\mathbb{G}_{n, n+1}$ acts diagonally on $S\left(\mathbf{F}_{n}, m\right) \otimes_{s, E_{n}^{0}} \mathbb{B}_{n}^{0}$, where the action on $S\left(\mathbf{F}_{n}, m\right)$ is given through the projection $\mathbb{G}_{n, n+1} \rightarrow \mathbb{G}_{n}$.

Lemma 5.6. The isomorphism $S\left(\mathbf{G}_{\mathbb{B}}, m, 0\right) \cong S\left(\mathbf{F}_{n}, m\right) \otimes_{s, E_{n}^{0}} \mathbb{B}_{n}^{0}$ respects the actions of $\mathbb{G}_{n, n+1}$ for any $m \geqslant 0$.

Proof. By diagram (7) and the isomorphism $\theta: \mathbf{G}_{\mathbb{B}}^{0} \cong \mathbf{H}_{\mathbb{B}}$, we obtain $\rho_{\mathbf{H}}(g) \circ \theta=$ $\theta \circ \rho_{\mathbf{G}}(g)^{0}$ for $g \in \mathbb{G}_{n, n+1}$. The lemma follows from the fact that $\rho_{\mathbf{H}}(g)$ is the identity map for any $g \in \mathbb{G}_{n+1}$.

The following lemma is obtained in the same way as Lemma 5.6.

Lemma 5.7. The map $\operatorname{inc}(m, r): S\left(\mathbf{F}_{n}, m-r\right) \rightarrow S\left(\mathbf{G}_{\mathbb{B}}, m, r\right)$ is $\mathbb{G}_{n, n+1}$-equivariant for any $0 \leqslant r \leqslant m$.

Lemma 5.8. The map $t_{m, r}^{\mathbb{B}}: \mathbb{B}_{n}^{0} \rightarrow S\left(\mathbf{G}_{\mathbb{B}}, m, r\right)$ is $\mathbb{G}_{n, n+1}$-equivariant for any $0 \leqslant$ $r \leqslant m$. 
Proof. We put $R=S\left(\mathbf{G}_{\mathbb{B}}, m, r\right)$. For $g \in \mathbb{G}_{n, n+1}$, we let $h=g \circ t_{m, r}^{\mathbb{B}}$ and $k=t_{m, r}^{\mathbb{B}} \circ g$. We can verify that $h \circ \mathrm{j}=k \circ \mathrm{j}$ and $h \circ$ inc $=k \circ$ inc. Recall that we have the fixed isomorphism $\theta: \mathbf{G}_{\mathbb{B}}^{0} \stackrel{\cong}{\rightrightarrows} \mathbf{H}_{\mathbb{B}}$. By Lemma 4.14, $\theta$ induces an isomorphism $\bar{\theta}:\left(\mathbf{G}_{R} / A_{R}\right)^{0} \stackrel{\cong}{\rightrightarrows}$ $\mathbf{H}_{R} / B_{R}$. By Lemma 4.2, the map $h$ corresponds to the isomorphism $g^{*} \bar{\theta}$. On the other hand, we have an isomorphism $g^{*} \theta: g^{*} \mathbf{G}_{\mathbb{B}}^{0} \cong g^{*} \mathbf{H}_{\mathbb{B}}$. This induces an isomorphism $\overline{g^{*} \theta}: g^{*} \mathbf{G}_{R}^{0} / g^{*} A_{R}^{0} \cong g^{*} \mathbf{H}_{R} / g^{*} B_{R}$. The map $k$ corresponds to the isomorphism $\overline{g^{*} \theta}$. Since $g^{*} \bar{\theta}=\overline{g^{*} \theta}$ under canonical identifications, we obtain $h=k$ by Lemma 4.2.

Corollary 5.9. The map $t_{m}^{\mathbb{B}}: \mathbb{B}_{n}^{0} \rightarrow S\left(\mathbf{F}_{n+1}, m\right) \otimes_{s, E_{n+1}^{0}} \mathbb{B}_{n}^{0}$ is $\mathbb{G}_{n, n+1}$-equivariant for any $m \geqslant 0$.

We suppose $\mathbb{G}_{n, n+1}$ acts on $\mathcal{S}_{n+1}$ through the projection $\mathbb{G}_{n, n+1} \rightarrow \mathbb{G}_{n+1}$.

Theorem 5.10. The profinite group $\mathbb{G}_{n, n+1}$ acts on $\mathcal{S}_{\mathbb{B}}$ and the map ch: $\mathcal{S}_{n+1} \rightarrow \mathcal{S}_{\mathbb{B}}$ is $\mathbb{G}_{n, n+1}$-equivariant.

Proof. This follows from Lemma 5.3 and Corollary 5.9.

\subsection{The graded Hopf algebroid $\mathcal{S}_{\mathbb{B}}^{0}$ without antipode}

We let $s_{m}=s_{m, 0}^{\mathbb{B}}: \mathbb{B}_{n}^{0} \rightarrow S\left(\mathbf{G}_{\mathbb{B}}, m, 0\right)$ be the inclusion, and we let $t_{m}=t_{m, 0}^{\mathbb{B}}: \mathbb{B}_{n}^{0} \rightarrow$ $S\left(\mathbf{G}_{\mathbb{B}}, m, 0\right)$ for $m \geqslant 0$. We let $i: S(\mathbf{G}, 0,0) \rightarrow \mathbb{B}_{n}^{0}$ be the canonical isomorphism. By Lemma 4.17, we have a map

$$
c_{m^{\prime}, m}=c_{m^{\prime}, m, 0,0}^{\mathbb{B}}: S\left(\mathbf{G}_{\mathbb{B}}, m+m^{\prime}, 0\right) \longrightarrow S\left(\mathbf{G}_{\mathbb{B}}, m^{\prime}, 0\right) \underset{s, \mathbb{B}_{n}^{0}, t}{\otimes} S\left(\mathbf{G}_{\mathbb{B}}, m, 0\right),
$$

for $m, m^{\prime} \geqslant 0$. Note that $S\left(\mathbf{F}_{n}, m^{\prime}\right) \otimes_{s, E_{n}^{0}, t} S\left(\mathbf{F}_{n}, m\right) \otimes_{s, E_{n}^{0}} \mathbb{B}_{n}^{0}$ is isomorphic to the target of the map $c_{m^{\prime}, m}$. We can identify $c_{m, m^{\prime}, 0,0}^{\mathbb{B}}$ with $c_{m, m^{\prime}}^{E_{n}^{n}} \otimes \operatorname{id}_{\mathbb{B}_{n}^{0}}$ by Lemma 4.18. By Proposition 4.19, we can see that the maps $t_{m, 0}^{\mathbb{B}}: \mathbb{B}_{n}^{0} \rightarrow S\left(\mathbf{F}_{n}, m\right) \otimes_{s, E_{n}^{0}} \mathbb{B}_{n}^{0}$ for $m \geqslant 0$ make $\mathbb{B}_{n}^{0}$ into a commutative $\mathcal{S}_{n}$-comodule algebra.

The action of $\mathbb{G}_{n, n+1}$ on $S\left(\mathbf{G}_{\mathbb{B}}, m\right)$ restricts to an action on $S\left(\mathbf{G}_{\mathbb{B}}, m, 0\right)$ and the map $t_{m}: \mathbb{B}_{n}^{0} \rightarrow S\left(\mathbf{G}_{\mathbb{B}}, m, 0\right)$ is $\mathbb{G}_{n, n+1}$-equivariant for any $m \geqslant 0$ by Lemma 5.8 . We suppose that $\mathbb{G}_{n, n+1}$ acts on $\mathcal{S}_{n}$ through the projection $\mathbb{G}_{n, n+1} \rightarrow \mathbb{G}_{n}$. By Lemmas 4.10 and 5.3 , we obtain the following theorem.

Theorem 5.11. The data $\mathcal{S}_{\mathbb{B}}^{0}=\left(\mathbb{B}_{n}^{0},\left\{S\left(\mathbf{G}_{\mathbb{B}}, m, 0\right)\right\}, s, t, i, c\right)$ is a graded Hopf algebroid without antipode. The map inc induces a map inc: $\mathcal{S}_{n} \rightarrow \mathcal{S}_{\mathbb{B}}^{0}$ of graded Hopf algebroids without antipode. The group $\mathbb{G}_{n, n+1}$ acts on $\mathcal{S}_{\mathbb{B}}^{0}$ and the map inc: $\mathcal{S}_{n} \rightarrow \mathcal{S}_{\mathbb{B}}^{0}$ is $\mathbb{G}_{n, n+1}$-equivariant.

The projections $p_{m, 0}: S\left(\mathbf{G}_{\mathbb{B}}, m\right) \rightarrow S\left(\mathbf{G}_{\mathbb{B}}, m, 0\right)$ for $m \geqslant 0$ induce a map $p_{0}: \mathcal{S}_{\mathbb{B}} \rightarrow$ $\mathcal{S}_{\mathbb{B}}^{0}$ of graded Hopf algebroids without antipode. Since $p_{m, 0}$ is $\mathbb{G}_{n, n+1}$-equivariant, we see that the map $p_{0}: \mathcal{S}_{\mathbb{B}} \rightarrow \mathcal{S}_{\mathbb{B}}^{0}$ is $\mathbb{G}_{n, n+1}$-equivariant.

Let $\psi_{M, m}: M \rightarrow S\left(\mathbf{G}_{\mathbb{B}}, m\right) \otimes_{s, \mathbb{B}_{n}^{0}} M$ for $m \geqslant 0$ be the comodule structure map for an $\mathcal{S}_{\mathbb{B}}$-comodule $M$. The composition $\left(p_{m, 0} \otimes \operatorname{id}_{M}\right) \circ \psi_{M, m}: M \rightarrow S\left(\mathbf{G}_{\mathbb{B}}, m, 0\right) \otimes_{s, \mathbb{B}_{n}^{0}}$ $M$ gives $M$ an $\mathcal{S}_{\mathbb{B}}^{0}$-comodule structure. Furthermore, if $M \in \mathbb{G}_{n, n+1}$-Comod $\left(\mathcal{S}_{\mathbb{B}}\right)$, the $\operatorname{map}\left(p_{m, 0} \otimes \operatorname{id}_{M}\right) \circ \psi_{M, m}$ is $\mathbb{G}_{n, n+1}$-equivariant for all $m \geqslant 0$. Hence we obtain a 
functor

$$
P_{0}: \mathbb{G}_{n, n+1}-\operatorname{Comod}\left(\mathcal{S}_{\mathbb{B}}\right) \longrightarrow \mathbb{G}_{n, n+1}-\operatorname{Comod}\left(\mathcal{S}_{\mathbb{B}}^{0}\right) .
$$

This functor restricts to a functor

$$
P_{0}: \mathbb{G}_{n, n+1} \text {-ComodAlg }\left(\mathcal{S}_{\mathbb{B}}\right) \longrightarrow \mathbb{G}_{n, n+1} \text { - } \operatorname{ComodAlg}\left(\mathcal{S}_{\mathbb{B}}^{0}\right) .
$$

\subsection{Comparison of comodules with compatible action}

In this subsection, we construct a comparison functor and prove our first main theorem.

Proposition 5.12. There exists an adjoint pair of functors

$$
L_{n+1}: \mathbb{G}_{n+1}-\operatorname{Comod}\left(\mathcal{S}_{n+1}\right) \leftrightarrows \mathbb{G}_{n, n+1}-\operatorname{Comod}\left(\mathcal{S}_{\mathbb{B}}\right): F_{n+1},
$$

where the left adjoint $L_{n+1}$ is the extension of scalars functor and the right adjoint $F_{n+1}$ is given by $F_{n+1}(N)=N^{\mathbb{G}_{n}}$ for $N \in \mathbb{G}_{n, n+1}-\operatorname{Comod}\left(\mathcal{S}_{\mathbb{B}}\right)$. This adjoint pair induces an adjoint pair of functors

$$
L_{n+1}: \mathbb{G}_{n+1} \text {-ComodAlg }\left(\mathcal{S}_{n+1}\right) \leftrightarrows \mathbb{G}_{n, n+1} \text {-ComodAlg }\left(\mathcal{S}_{\mathbb{B}}\right): F_{n+1}
$$

Proof. This follows from Proposition 5.4, Theorem 5.10, and the fact that $S\left(\mathbf{F}_{n+1}, m\right)$ is a free module over $E_{n+1}^{0}$ via $s_{m}$ for all $m \geqslant 0$.

In the same way we obtain the following proposition.

Proposition 5.13. There is an adjoint pair of functors

$$
L_{n}: \mathbb{G}_{n}-\operatorname{Comod}\left(\mathcal{S}_{n}\right) \leftrightarrows \mathbb{G}_{n, n+1}-\operatorname{Comod}\left(\mathcal{S}_{\mathbb{B}}^{0}\right): F_{n}
$$

where the left adjoint $L_{n}$ is the extension of scalars functor and the right adjoint $F_{n}$ is given by $F_{n}(N)=N^{\mathbb{G}_{n+1}}$ for $N \in \mathbb{G}_{n, n+1}$-Comod $\left(\mathcal{S}_{\mathbb{B}}^{0}\right)$. This induces an adjoint pair of functors

$$
L_{n}: \mathbb{G}_{n}-\operatorname{ComodAlg}\left(\mathcal{S}_{n}\right) \leftrightarrows \mathbb{G}_{n, n+1}-\operatorname{ComodAlg}\left(\mathcal{S}_{\mathbb{B}}^{0}\right): F_{n}
$$

Proof. This follows from Proposition 5.4, Theorem 5.11 and the fact that $S\left(\mathbf{F}_{n}, m\right)$ is a free module over $E_{n}^{0}$ via $s_{m}$ for all $m \geqslant 0$.

The following is our first main theorem.

Theorem 5.14. There is a functor

$$
C: \mathbb{G}_{n+1}-\operatorname{Comod}\left(\mathcal{S}_{n+1}\right) \longrightarrow \mathbb{G}_{n}-\operatorname{Comod}\left(\mathcal{S}_{n}\right)
$$

given by

$$
C(M)=\left(\mathbb{B}_{n}^{0} \otimes_{E_{n+1}^{0}} M\right)^{\mathbb{G}_{n+1}},
$$

for $M \in \mathbb{G}_{n+1}$-Comod $\left(\mathcal{S}_{n+1}\right)$. Furthermore, this functor induces a functor

$$
C: \mathbb{G}_{n+1} \text {-ComodAlg }\left(\mathcal{S}_{n+1}\right) \longrightarrow \mathbb{G}_{n} \text {-ComodAlg }\left(\mathcal{S}_{n}\right) \text {. }
$$

Proof. We have the functors $L_{n+1}: \mathbb{G}_{n+1}-\operatorname{Comod}\left(\mathcal{S}_{n+1}\right) \rightarrow \mathbb{G}_{n, n+1}-\operatorname{Comod}\left(\mathcal{S}_{\mathbb{B}}\right)$ and $F_{n}: \mathbb{G}_{n, n+1}-\operatorname{Comod}\left(\mathcal{S}_{\mathbb{B}}^{0}\right) \rightarrow \mathbb{G}_{n}-\operatorname{Comod}\left(\mathcal{S}_{n}\right)$ by Propositions 5.12 and 5.13. We obtain the desired functor $C$ as the composition $F_{n} P_{0} L_{n+1}$. 


\subsection{Morava $E$-theories with power operations}

Let $\mathcal{S}$ be the stable homotopy category. For a spectrum $Z$, we denote by $\Lambda(Z)$ the full subcategory of the over category $(\mathcal{S} \downarrow Z)$ whose objects are maps $Z_{\lambda} \rightarrow Z$ with $Z_{\lambda}$ finite. Let $X$ be a $C W$-complex and let $\left\{X_{\alpha}\right\}$ be the filtered system of all the finite subcomplexes of $X$. We denote by $\left\{\Sigma_{+}^{\infty} X_{\alpha}\right\}$ the filtered system in $\mathcal{S}$ obtained by applying the functor $\Sigma_{+}^{\infty}(-)$ to $\left\{X_{\alpha}\right\}$. Note that we can regard $\left\{\Sigma_{+}^{\infty} X_{\alpha}\right\}$ as a subcategory of $\Lambda\left(\Sigma_{+}^{\infty} X\right)$ and the inclusion functor from $\left\{\Sigma_{+}^{\infty} X_{\alpha}\right\}$ to $\Lambda\left(\Sigma_{+}^{\infty} X\right)$ is cofinal (see, for example, [8, Definition 2.3.8] for the definitions of a filtered category and a cofinal functor).

Since $\mathbb{B}_{n}^{0}$ is a complete Noetherian local ring, $\mathbb{B}_{n}^{0}$ is a linearly compact $\mathbb{B}_{n}^{0}$-module with respect to the $I_{n}$-adic topology, where $I_{n}=\left(p, w_{1}, \ldots, w_{n-1}\right)$ is the maximal ideal of $\mathbb{B}_{n}^{0}$ (see [8, Definition 2.3.13] for the definition of a linearly compact module). Since $\mathbb{B}_{n}$ is an even-periodic commutative ring spectrum with $\mathbb{B}_{n}^{0}$ Noetherian and linearly compact, there is a natural isomorphism

$$
\mathbb{B}_{n}^{0}(X) \cong \lim \mathbb{B}_{n}^{0}\left(Z_{\lambda}\right)
$$

by [16, Lemma 3.4], where the limit is taken over $\left(Z_{\lambda} \rightarrow \Sigma_{+}^{\infty} X\right) \in \Lambda\left(\Sigma_{+}^{\infty} X\right)$. Since the inclusion functor from $\left\{\Sigma_{+}^{\infty} X_{\alpha}\right\}$ to $\Lambda\left(\Sigma_{+}^{\infty} X\right)$ is cofinal, we obtain a natural isomorphism

$$
\mathbb{B}_{n}^{0}(X) \cong \lim _{\alpha} \mathbb{B}_{n}^{0}\left(X_{\alpha}\right)
$$

If $X$ is a finite complex, then we have a natural isomorphism $\mathbb{B}_{n}^{0}(X) \cong \mathbb{B}_{n}^{0} \otimes_{E_{n+1}^{0}}$ $E_{n+1}^{0}(X)$. The natural commutative $\mathcal{S}_{n+1}$-comodule algebra structure with compatible $\mathbb{G}_{n+1}$-action on $E_{n+1}^{0}(X)$ produces a natural commutative $\mathcal{S}_{\mathbb{B}}$-comodule algebra structure on $\mathbb{B}_{n}^{0}(X)$ with compatible $\mathbb{G}_{n, n+1}$-action by Proposition 5.12 . Using isomorphism (8), we obtain a natural commutative $\mathcal{S}_{\mathbb{B}}$-comodule algebra structure on $\mathbb{B}_{n}^{0}(X)$ with compatible $\mathbb{G}_{n, n+1}$-action

$$
\mathbb{B}_{n}^{0}(X) \in \mathbb{G}_{n, n+1} \text {-ComodAlg }\left(\mathcal{S}_{\mathbb{B}}\right),
$$

for any space $X$ since $S\left(\mathbf{G}_{\mathbb{B}}, m\right)$ is a finitely generated free $\mathbb{B}_{n}^{0}$-module via $s_{m}^{\mathbb{B}}$ for all $m \geqslant 0$. Let $\Psi_{m}^{\mathbb{B}}: \mathbb{B}_{n}^{0}(X) \rightarrow S\left(\mathbf{G}_{\mathbb{B}}, m\right) \otimes_{s, \mathbb{B}_{n}^{0}} \mathbb{B}_{n}^{0}(X)$ be the comodule structure maps, and we set $\Psi_{m, 0}^{\mathbb{B}}=\left(p_{m, 0} \otimes 1\right) \circ \Psi_{m}^{\mathbb{B}}: \mathbb{B}_{n}^{0}(X) \rightarrow S\left(\mathbf{G}_{\mathbb{B}}, m, 0\right) \otimes_{s, \mathbb{B}_{n}^{0}} \mathbb{B}_{n}^{0}(X)$ for $m \geqslant 0$.

Lemma 5.15. There is a natural commutative diagram

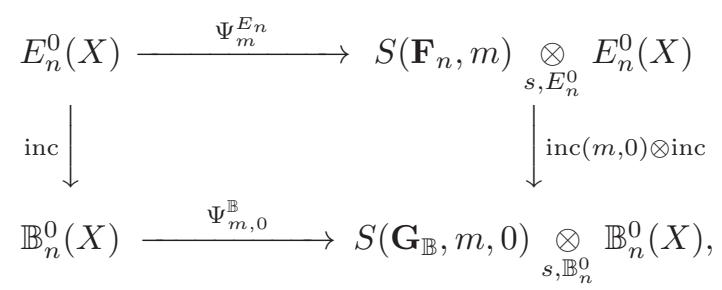

for any space $X$ and any $m \geqslant 0$.

Proof. By [2, Proposition 3.7], an unstable ring operation between Landweber exact cohomology theories is determined by its values on the one-point space and the infinite complex projective space $\mathbb{C P}^{\infty}$. Hence it is sufficient to show that the diagram is 
commutative when $X$ is the one-point space and when $X$ is $\mathbb{C P}^{\infty}$. If $X$ is the one-point space, then the diagram is commutative since $t_{m, 0}^{\mathbb{B}} \circ \mathrm{ch}=\operatorname{ch}(m, 0) \circ t_{m}^{E_{n+1}}$ and $t_{m, 0}^{\mathbb{B}} \circ$ inc $=\operatorname{inc}(m, 0) \circ t_{m}^{E_{n}}$. If $X=\mathbb{C P}^{\infty}$, then we see that the diagram is commutative by commutative diagram (6).

Proposition 5.16. The natural map inc: $E_{n}^{0}(X) \rightarrow \mathbb{B}_{n}^{0}(X)$ induces an isomorphism

$$
E_{n}^{0}(X) \cong F_{n} P_{0}\left(\mathbb{B}_{n}^{0}(X)\right),
$$

of commutative $\mathcal{S}_{n}$-comodule algebras with compatible $\mathbb{G}_{n}$-action for any space $X$.

Proof. The map inc: $E_{n}^{0}(X) \rightarrow \mathbb{B}_{n}^{0}(X)$ induces an isomorphism $E_{n}^{0}(X) \stackrel{\cong}{\rightarrow} F_{n}\left(\mathbb{B}_{n}^{0}(X)\right)$ in $\mathbb{G}_{n}-\mathrm{CAlg}\left(E_{n}^{0}\right)$ by Lemma 5.2. By Lemma 5.15 , we see that this is an isomorphism of commutative $\mathcal{S}_{n}$-comodule algebras with compatible $\mathbb{G}_{n}$-action.

Theorem 5.17. For any finite complex $X$, there is a natural isomorphism

$$
E_{n}^{0}(X) \cong C\left(E_{n+1}^{0}(X)\right),
$$

of commutative $\mathcal{S}_{n}$-comodule algebras with compatible $\mathbb{G}_{n}$-action.

Proof. We have a natural isomorphism $\mathbb{B}_{n}^{0}(X) \cong L_{n+1}\left(E_{n+1}^{0}(X)\right)$ of commutative $\mathcal{S}_{\mathbb{B}^{-}}$ comodule algebras with compatible $\mathbb{G}_{n, n+1}$-action when $X$ is a finite complex. The theorem follows from Proposition 5.16.

\section{Acknowledgments}

The author would like to thank the anonymous referee for careful reading of the manuscript and many helpful suggestions.

\section{References}

[1] M. Ando, Isogenies of formal group laws and power operations in the cohomology theories $E_{n}$, Duke Math. J. 79 (1995), no. 2, 423-485.

[2] M. Ando, Power operations in elliptic cohomology and representations of loop groups, Trans. Amer. Math. Soc. 352 (2000), no. 12, 5619-5666.

[3] M. Ando, M.J. Hopkins and N.P. Strickland, The sigma orientation is an $H_{\infty}$ map, Amer. J. Math. 126 (2004), no. 2, 247-334.

[4] E.S. Devinatz, Morava's change of rings theorem, The Čech Centennial (Boston, MA, 1993), 83-118, Contemp. Math. 181, Amer. Math. Soc., Providence, RI, 1995.

[5] E.S. Devinatz, A Lyndon-Hochschild-Serre spectral sequence for certain homotopy fixed point spectra, Trans. Amer. Math. Soc. 357 (2005), no. 1, 129-150.

[6] P.G. Goerss and M.J. Hopkins, Moduli spaces of commutative ring spectra, Structured ring spectra, London Math. Soc. Lecture Note Ser. 315 (2004), $151-200$.

[7] C. Greither, Cyclic Galois Extensions of Commutative Rings, Lecture Notes in Math. 1534, Springer-Verlag, Berlin, 1992. 
[8] M. Hovey, J.H. Palmieri and N.P. Strickland, Axiomatic Stable Homotopy Theory, Mem. Amer. Math. Soc. 128 (1997), no. 610.

[9] N.M. Katz and B. Mazur, Arithmetic Moduli of Elliptic Curves, Ann. of Math. Stud. 108, Princeton University Press, Princeton, NJ, 1985.

[10] M. Rapoport and Th. Zink, Period Spaces for $p$-Divisible Groups, Ann. of Math. Stud. 141, Princeton University Press, Princeton, NJ, 1996.

[11] D.C. Ravenel, Complex Cobordism and Stable Homotopy Groups of Spheres, Pure Appl. Math. 121, Academic Press, Inc., Orlando, FL, 1986.

[12] C. Rezk, The congruence criterion for power operations in Morava E-theory, Homology Homotopy Appl. 11 (2009), no. 2, 327-379.

[13] N.P. Strickland, Finite subgroups of formal groups, J. Pure Appl. Algebra 121 (1997), no. 2, 161-208.

[14] N.P. Strickland, Morava E-theory of symmetric groups, Topology 37 (1998), no. 4, 757-779.

[15] T. Torii, On degeneration of formal group laws and application to stable homotopy theory, Amer. J. Math. 125 (2003), 1037-1077.

[16] T. Torii, Comparison of Morava E-theories, Math. Z. 266 (2010), no. 4, 933951.

[17] T. Torii, On $E_{\infty}$-structure of the generalized Chern character, Bull. Lond. Math. Soc. 42 (2010), no. 4, 680-690.

[18] T. Torii, HKR characters, $p$-divisible groups and the generalized Chern character, Trans. Amer. Math. Soc. 362 (2010), no. 11, 6159-6181.

[19] T. Torii, Hecke operators in Morava E-theories of different heights, Preprint.

Takeshi Torii torii@math.okayama-u.ac.jp

Department of Mathematics, Okayama University, Okayama, 700-8530, Japan 